Review Article

\title{
Level Spectroscopy in Quantum Antiferromagnets Using Monte Carlo Simulations
}

\author{
ARNAB SEN* \\ Department of Theoretical Physics, Indian Association for the Cultivation of Science, Jadavpur, Kolkata \\ 700 032, India
}

(Received on 16 October 2017; Revised on 01 January 2018; Accepted on 14 March 2018)

\begin{abstract}
The low-energy spectrum of antiferromagnets reveal valuable information about the nature of the phase or phase transition in such systems. I review the recent works done in collaboration with Suwa and Sandvik (Phys Rev B 92195145 (2015), Phys Rev B 94144416 (2016)) on how to probe the dispersion of the excitations in a variety of SU(2) symmetric $S=1 / 2$ spin systems using quantum Monte-Carlo methods. Various applications are discussed in both one and two dimensions, which include probing the critical excitations for conventional and unconventional quantum critical points. In the latter case, the excitation spectrum is highly unusual and has additional gapless modes and possible emergent symmetries which are not present otherwise.
\end{abstract}

Keywords: Quantum Antiferromagnets; Deconfined Quantum Critical Points; Spin Waves; Spinons; Quantum Monte Carlo Methods

\section{Introduction}

At low temperatures, the magnetic degrees of freedom in a Mott insulator can be described by integrating out the high-energy charge fluctuation processes which then usually results in an "effective" spin Hamiltonian of the following form [1]:

$$
H=J \sum_{\langle i j\rangle} \mathbf{S}_{i} \cdot \mathbf{S}_{j}+g H^{\prime}
$$

where $J(>0)$ is the (antiferromagnetic) Heisenberg exchange constant, $\mathbf{S}$ is the appropriate quantummechanical operator that describes the magnetic moment carried by the ions in the Mott insulator, and $\langle i j\rangle$ denote nearest neighbour sites $i, j$ on the lattice. $H^{\prime}$ in Eq. 1 represent possible competing short-ranged interactions (such as interactions beyond nearest neighbors, ring exchange terms etc.) which, like the Heisenberg exchange terms, preserve all the lattice symmetries and their relative importance is controlled by the parameter $g$. The physics of the family of models described by Eq. 1 is very rich and is determined by several factors that include the size of the $\operatorname{spin} \mathbf{S}(S=1 / 2,1,3 / 2, \cdots)$, nature of the additional competing terms denoted by $H^{\prime}$ in Eq. 1 , and the dimensionality and geometry of the underlying lattice.

Let us consider the extreme quantum limit of $S=1 / 2$ spins and ask for the possible ground states of $H$ for the two-dimensional square lattice. The magnetically ordered Néel state (Fig. 1) represents the simplest possible ground state. Classically, the ground state has $S_{\mathrm{r}}^{z}=+1 / 2$ (with the $z$ axis being arbitrary) on one sublattice and $S_{\mathrm{r}}^{z}=-1 / 2$ for the other sublattice. However, the staggered magnetization, which acts as the order parameter for the Néel state, does not commute with the Hamiltonian when $\mathrm{S}$ is finite and the simple-minded classical ground state is not the true quantum ground state. Does Néel order survive in the quantum ground state or is the true ground state for $S=1 / 2$ spins without any longranged magnetic order? It has been rigorously shown [2] that for the nearest-neighbour Heisenberg antiferromagnet on a $d$-dimensional hypercubic lattice, the ground state has Néel order for all $S$ for $d \geq 3$ and for $S \geq 1$ when $d=2$. The interesting case of $S=1 / 2$

*Authorfor Correspondence: E-mail: tpars@iacs.res.in 

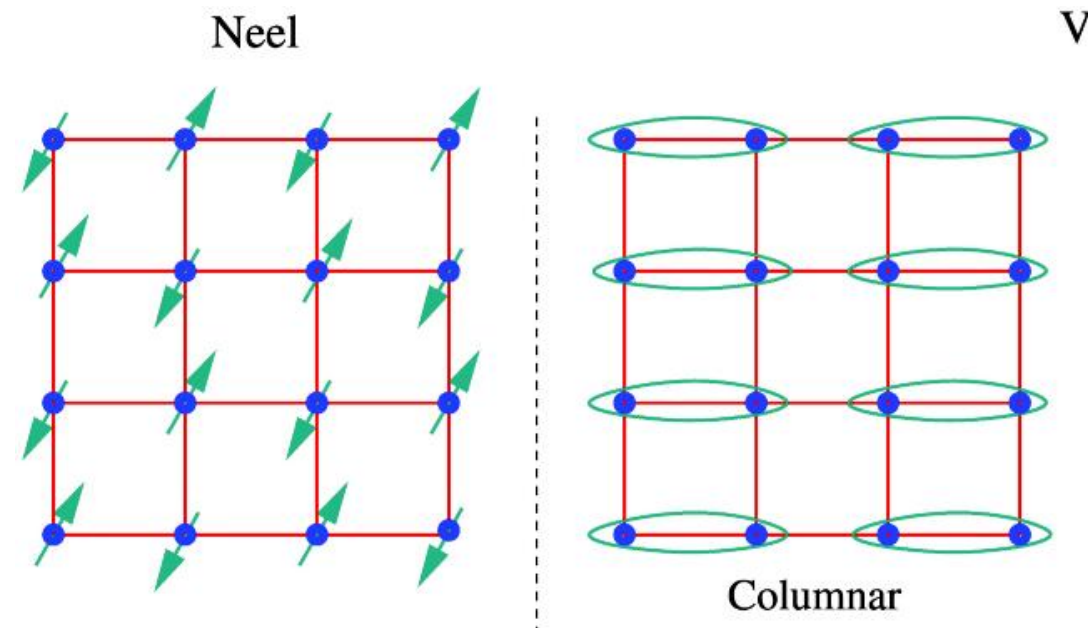

\section{Valence Bond Crystal}
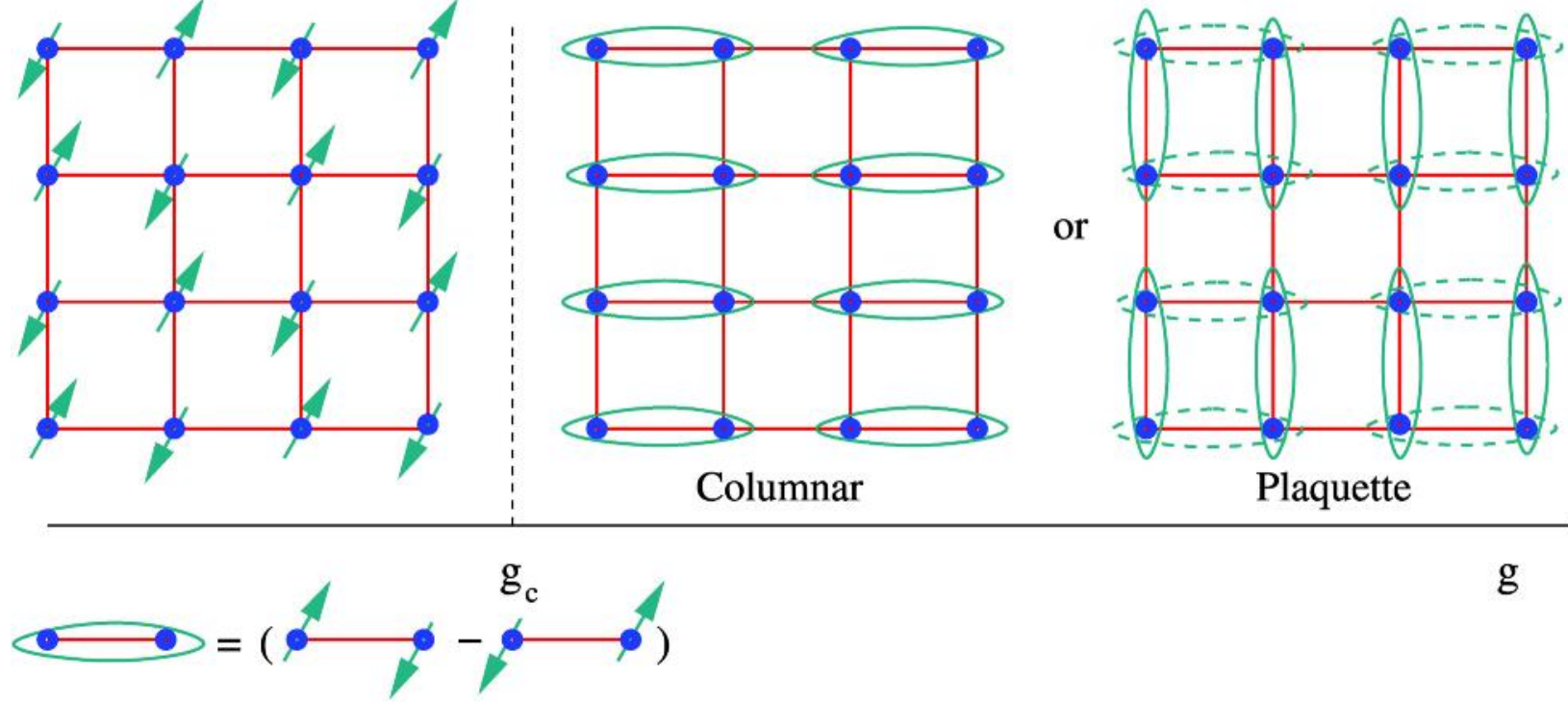

Plaquette

Fig. 1: Some possible ground states of the square lattice $S=1 / 2$ quantum antiferromagnet. The coupling $g$ controls the strength of the competing terms that can destabilize the magnetically ordered Néel state and drive a phase transition to a different ground state like a valence bond crystal (VBC). Both the Néel and the VBC state spontaneously break symmetries of the underlying interactions

on the square lattice remains out of reach of these rigorous methods. However, Monte Carlo simulations show [3] that the ground state continues to have longrange Néel order when $S=1 / 2$, with the order parameter reduced to about $40 \%$ of its classical value. What about other possible ground states of $H$ (when $g \neq 0$ ) such that these states do not break spin rotation symmetry? As a concrete example, consider the $J_{1}-$ $J_{2}$ model on the square lattice, where in addition to the nearest neighbour interaction $J_{1}$, there is also a next nearest nearest neighbour interaction $J_{2}$ (thus $g$ $=J_{2} / J_{1}$ in Eq. 1 for this model). The classical limit of this model $(S \rightarrow \infty)$ has collinear Néel order for all $g$ $=J_{2} / J_{1}$. For very small $g$, Néel order survives in the quantum ground state for $S=1 / 2$ as well. However, numerical and series expansion studies [4] for $S=1 /$ 2 have shown that this model loses the magnetic order around $g \approx 0.5$ and spin rotation symmetry is restored. The ground state breaks lattice symmetry instead (eventually a stripe antiferromagnetic state is stabilized for $g$ larger than $g \approx 0.6$ ). The nature of this quantum phase transition has remained controversial despite several numerical studies, and we refer the readers to Ref. 5 for a very recent work on this issue and background literature. More generally, such quantum paramagnetic states can be broadly divided into two classes. Firstly there are states that can be described as "valence bond crystal" (VBC) states. In a simple caricature of such a state, each spin forms a singlet with one of its neighbouring spins resulting in an ordered pattern of "valence bonds" (the singlets) (see Fig. 1). For $S=1 / 2$ systems on a square lattice, such states necessarily break lattice translational symmetry and the ground state is four-fold degenerate. The symmetry can be broken in (at least) two different ways, leading to what is called columnar order and plaquette order (Fig. 1) respectively. In the plaquette state, singlet bonds resonate coherently between the two horizontal and vertical bonds of the elementary square plaquettes on the lattice (shown as dotted and undotted valence bonds in Fig. 1). Secondly, a class of more exotic paramagnetic states [6-10] is also possible: in these states the valence bond configurations resonate amongst each other and form a "spin liquid". The resulting state preserves all the global symmetries of the microscopic Hamiltonian and it is instead characterized by fractional spin-1/2 excitations (called spinons) and a non-trivial topological structure. Lastly, to complete the classification of known quantum paramagnetic states at zero 
temperature, a dimerized state that breaks no symmetries of the microscopic interactions can be stabilized for a lattice with two (or, a larger even number of) $S=1 / 2$ spins per unit cell by introducing a pattern of different Heisenberg couplings in such a manner that favours singlet formation on dimers (or, larger units of an even number of spins). Such dimerized states preserves all the symmetries of the interactions and are dubbed quantum-disordered states to distinguish them from VBC states (that spontaneously break lattice symmetries) or spin liquid states (that have fractional excitations and topological order). Both the Shastry Sutherland model [11] and the bilayer Heisenberg model [33] (the latter will be discussed in more detail later) are Hamiltonians where such quantum-disordered ground states are realized.

Quantum field theory techniques provide a very general framework for addressing low-energy properties of such interacting spin systems [13]. However, in many cases, while such an approach is really useful for the classification of possible phases on general grounds, several other details at the lattice level are important in deciding which phase is ultimately realized by a microscopic lattice Hamiltonian. Furthermore, any field theory has certain free parameters that have to be determined from a microscopic calculation to match its results (that apply at low-energy or equivalently at long distances) to an actual lattice model. Quantum Monte Carlo (QMC) methods provide an unbiased route for calculating properties of spin models that do not suffer from the notorious "sign problem" [14] for system sizes that typically far exceed other numerical methods like exact diagonalization. However, unlike exact diagonalization which gives access to all possible observables, QMC simulations typically allow for calculations of quantities like energy, uniform magnetic susceptibility, spin stiffness, and certain kinds of imaginary-timedependent correlation functions. Ageneral introduction to computational methods in quantum spin systems can be found in Ref. 15.

\section{A. Outline}

In this short review, we will discuss QMC methods developed in Refs. 16, 17 to effciently calculate energy gaps in different spin $(S)$ and momentum $(\mathbf{k})$ sectors for certain $S U(2)$ symmetric spin-1/2 models in one and two dimensions which allows for the calculation of the dispersion relation and the velocity of the propagating singlet $(S=0)$ and triplet $(S=1)$ excitations for ordered, disordered and critical antiferromagnets.

The rest of the paper is organized as follows: In Sec. II, we discuss calculations of imaginary-time correlation functions within a ground-state projector QMC method to extract the lowest momentumdependent gaps from their long-time behaviours. We compare our results with exact solutions for small Heisenberg chains as well as with the rigorously known velocity of the low-energy propagating modes in the thermodynamic limit. In Sec. III, we discuss the determination of the velocity based on an indirect but powerful approach of winding numbers and demonstrate the method using the Heisenberg chain example. For the winding numbers calculations, we require QMC at finite temperatures which is done using the stochastic series expansion (SSE) QMC method. In Sec. IV, we present results for the standard two-dimensional Heisenberg model on the square lattice. We also show that the scaling of the triplet gap at $\mathbf{k}=(\pi, \pi)$ is entirely consistent with quantumrotor states that carry spin $S=1$. In Sec. V, we show results for the critical and disordered Heisenberg bilayer systems. In Sec. VI, we show results for the level spectrum of the $J-Q$ model at its deconfined quantum critical point which reveals a highly unusual excitation spectrum with gapless critical $S=0$ and $S$ $=1$ excitations at $\mathbf{k}=(0,0),(\pi, 0),(0, \pi)$ and $(\pi, \pi)$ where all these points are characterized by linear dispersion with a common velocity. Moreover, we show signatures in the excitation spectrum that point towards an emergent $\mathrm{SO}(5)$ symmetry at the critical point. Both these features highlight the unconventional nature of this quantum critical point. Sec. II, Sec. III, Sec. IV and Sec. V are based on Ref. 16 and Sec. VI is based on Ref. [17]. Finally, we conclude in Sec. VII.

\section{Momentum-Dependent Spin Gaps}

Here we discuss how to use zero temperature ( $T=$ $0)$ projector QMC methods to calculate imaginarytime correlation functions which are then used to extract the singlet $(S=0)$ and triplet $(S=1)$ gaps with respect to the ground state energy as a function of momentum k. The projector QMC can be carried out in two ways. Applying the imaginary-time evolution 
operator $e^{-\beta H}$ to a trial state leads to the ground state when $\beta \rightarrow \infty$. Alternatively, one can use a high power $H^{m}$ of the Hamiltonian and reach the ground state when $m \rightarrow \infty$. We will explain the use of the latter approach here (mainly because it is simpler to apply) and show ways of analyzing the correlation functions to extract the lowest excitation energy at each $\mathbf{k}$. For an alternate approach to extract the gaps in the singlet and triplet sectors using a generalized moment method, where a series of moments of the imaginary-time correlation functions is systematically extrapolated to extract the smallest gap, we refer to readers to Refs. $16,23$.

\section{A. H-power Projection}

This QMC approach is based on projection with a suffciently high power of the Hamiltonian $H$ on a trial state $\left|\psi_{t}\right\rangle$. The process can be expressed in the energy eigenbasis of $H$, leading to the following expression for the dependence on $m$ :

$$
(-H)^{m}\left|\psi_{t}\right\rangle=c_{0}\left(-E_{0}\right)^{m}\left[|0\rangle+\sum_{n-1}^{\Lambda} \frac{c_{n}}{c_{0}}\left(\frac{E_{n}}{E_{0}}\right)|n\rangle\right] .
$$

Here $|n\rangle, n=0, \cdots, \Lambda-1$ are the energy eigenstates of $H$ and $E_{0}$ is assumed to be negative, with its absolute value $\left|E_{0}\right|$ being the largest in magnitude of all the energies (this can always be achieved by adding a suitable constant to the Hamiltonian). Then, if the expansion coefficient $c_{0} \neq$ 0 , we have $(-H)^{m}\left|\psi_{t}\right\rangle \propto|0\rangle$ for large $m$, and the expectation value of an operator $O$ at $T=0$ can be written as

$$
\langle O\rangle=\frac{\left\langle\psi_{t}\left|(-H)^{m} O(-H)^{m}\right| \psi_{t}\right\rangle}{\left\langle\psi_{t}\left|(-H)^{2 m}\right| \psi_{t}\right\rangle}
$$

For the class of SU(2) invariant spin models considered here, this form of the expectation value can be evaluated by importance-sampling, using a formulation of the projector QMC method in the nonorthogonal valence bond basis [18]. An efficient way of sampling the contributions to $(-H)^{2 m}$, very similar to "operator-loop" updates developed within the finiteT SSE method, [19] can be formulated in a combined space of spin components $\left(S^{z}\right)$ and valence bonds [20]. The details of the initial trial state are not crucial (apart from the fact that it should satisfy $S=0$ and $\mathbf{k}=0$ as these are also the conserved quantum numbers of the true ground state for the $\mathrm{SU}(2)$ symmetric antiferromagnetic spin models considered here on any finite lattice), as good convergence to the ground state is observed even if the state is not optimized [20].

\section{Imaginary-time Correlation Functions}

We consider correlation functions of the following form:

$$
C_{A}(t)=\frac{\left\langle 0\left|A^{\dagger}(-H)^{t} A\right| 0\right\rangle}{\left\langle 0\left|(-H)^{t}\right| 0\right\rangle}=\sum_{n=0}^{\Lambda-1}\left|\frac{E_{n}}{E_{0}}\right|^{t}|\langle n|A| 0\rangle|^{2}
$$

where $t$ is an integer which can be related to imaginary time [21] and we will loosely refer to it by this term. More precisely, $t / N$, where $N$ is the system size, is proportional to imaginary time $\tau$ in the sense of the standard Schrödinger time evolution operator $e^{-\tau H}$. From $C_{A}(t)$, we further define

$$
Q_{A}(t)=\frac{\left.\left\langle 0\left|A^{\dagger}(-H)^{t} A\right| 0\right\rangle\right|^{2}}{\left.\left\langle 0\left|(-H)^{t}\right| 0\right\rangle\right|^{2}},
$$

and note that $Q_{A}(0)=1$ and $Q_{A}(t \rightarrow \infty) \rightarrow 0$. For large $t$, we have

$$
Q_{A}(t)=\frac{|\langle 1|A| 0\rangle|^{2}}{\left\langle 0\left|A^{\dagger} A\right| 0\right\rangle-|\langle 0|A| 0\rangle|^{2}}\left(1-\frac{\Delta}{\left|E_{0}\right|}\right)^{t},
$$

where $\Delta=E_{1}-E_{0}$ is the energy gap between the first excited state (connected to the ground state by the operator $A$ ) and the ground state. To directly show the relationship between $t$ and imaginary time, we can introduce $\tau=t /\left|E_{0}\right|$, then write $E_{0}=N e_{0}$. Then, in the limit of large $N$, we have

$$
Q_{A}\left(\tau=t /\left|E_{0}\right|\right) \propto\left(1-\frac{\Delta}{N\left|e_{0}\right|}\right)^{N\left|e_{0}\right| \tau} \rightarrow e^{-\Delta \tau},
$$

which is the familiar form of the asymptotic decay of an imaginary-time dependent correlation function.

We can appropriately choose the operator A so that it excites the ground state $|0\rangle$ into a state with the desired quantum numbers. The ground state of the unfrustrated Hamiltonians considered here are total spin singlets with momentum $\mathbf{k}=0$ on finite lattices with an even number of spins and periodic boundary 
conditions (in one dimension only when the number $N$ of sites is a multiple of four-for other even $N$ the momentum is $\pi$ ). For triplet excitations, we can use the simple Fourier-transformed spin operator,

$$
A_{\mathrm{k}}=\mathscr{C}_{\mathrm{k}}=\frac{1}{\sqrt{N}} \sum_{\mathrm{r}} \mathrm{e}^{\mathrm{ik} \cdot \mathrm{r}} S_{\mathrm{r}}^{z}
$$

to create an $S=1$ state with momentum $\mathbf{k}$ and $S^{z}=0$ when acting on the ground state. For singlet excitations, we can use

$$
A_{\mathrm{k}}=\mathscr{C}_{\mathrm{k}}=\frac{1}{\sqrt{N}} \sum_{\mathrm{r}} \mathrm{e}^{\mathrm{ik} \cdot \mathrm{r}} S_{\mathrm{r}}^{z} S_{\mathrm{r}+\mathrm{e}}^{z},
$$

where e denotes an elementary unit vector of the (Bravais) lattice. Although $/ \mathrm{k}$ also creates $S=2$ excitations, a singlet has the lowest energy among the even- $S$ excitations in these systems. The following imaginary-time correlation

$$
\begin{aligned}
& C_{\mathrm{k}}(t)=\frac{\left\langle 0\left|A_{-\mathrm{k}}(-H)^{t} A_{\mathrm{k}}\right| 0\right\rangle}{\left\langle 0\left|(-H)^{t}\right| 0\right\rangle} \\
& =\frac{\left\langle\psi_{t}\left|(-H)^{2 m-p-t} A_{-\mathrm{k}}(-H)^{t} A_{\mathrm{k}}(-H)^{p}\right| \psi\right\rangle}{\left\langle\psi_{t}\left|(-H)^{2 m}\right| \psi_{t}\right\rangle}
\end{aligned}
$$

allows us to directly measure the (singlet) triplet excitation gap for $\left(A_{\mathrm{k}}=\mathscr{C}_{\mathrm{k}}\right) A_{\mathrm{k}}=\mathscr{C}_{\mathrm{k}}$ as a function of the momentum $\mathrm{k}$. The second line in the above equation explicitly shows the form used in the projector QMC calculations, where both $2 m-p-t$ and $p$ are assumed to be large enough to achieve projection to the ground state for the system sizes considered. In practice, we use projection powers $m=16 p_{r} N$ and, to achieve good ground-state convergence, require that $2 m-p-t$ and $p$ both are larger than $15 p_{r} N$, where typically $p_{r}=8$ (16 or higher in some cases). These choices are motivated by extensive tests indicating that no detectable systematical errors remain. The values of $t$ are restricted to be multiples of N/4 in our simulations. Since the correlation functions at the different $t$ values are measured in the same simulation, the $C_{\mathrm{k}}(t)$ data are correlated and measuring at shorter $t$ intervals does not significantly increase the amount of statistical information in the data set.

\section{Gap Extraction}

We use two different ways to extract the lowest singlet/triplet gap [16] from the correlation function $C_{\mathrm{k}}(t)$. Let us focus on the case of $A_{\mathrm{k}}=C_{\mathrm{k}}$ for simplicity. Since $\left\langle 0\left|A_{\mathrm{k}}\right| 0\right\rangle=0$ for $k \neq 0$ in that case, Eq. (5) reduces to

$$
Q_{\mathrm{k}}(t)=\frac{C_{\mathrm{k}}(t)}{C_{\mathrm{k}}(0)}
$$

In principle, the triplet gap can be extracted by monitoring the long-time behavior, given by the form (6). A systematic way to extract the gap without performing any curve fitting is to consider the ratio of $Q_{\mathrm{k}}(t)$ at two different times separated by some interval; e.g., by $N / 4$ operations:

$$
R_{\mathrm{k}}(t)=\left|E_{0}\right|\left(1-\left[\frac{Q_{\mathrm{k}}(t+N / 4)}{Q_{\mathrm{k}}(t)}\right]^{4 / N}\right) \text {. }
$$

$R_{\mathrm{k}}(t) \rightarrow(\mathscr{f})$ when $\mathrm{t}$ is large enough, which follows from the long-time behavior of $Q_{\mathrm{k}}(t)$ in Eq. (6). However, it is not always possible to reach perfect convergence of this gap estimate for all $\mathrm{k}$, because the relative statistical errors often become too large already for moderately large $t$.

We thus use another method to estimate the value of the gap which is based on the entire available set of correlation functions. It is clear from Eq. (4) that one can define a positive-definite spectral function $A_{\mathrm{k}}(\omega)$ to fit the normalized imaginary-time correlation $Q_{\mathrm{k}}(t)$ as

$$
Q_{\mathrm{k}}(t)=\int_{0}^{\infty} d \omega A_{\mathrm{k}}(\omega)\left(1-\frac{\omega}{\left|E_{0}\right|}\right)^{t} .
$$

This is just the analogue for the $H$-power evolution of the standard form,

$$
G_{\mathrm{k}}(\tau)=\int_{0}^{\infty} d \omega S_{\mathrm{k}}(\omega) \mathrm{e}^{-\omega r},
$$

relating the imaginary-time Schrödinger evolved correlation function

$$
G_{\mathrm{k}}(\tau)=\left\langle 0\left|S_{-\mathrm{k}}^{z}(\tau) S_{\mathrm{k}}^{z}(0)\right| 0\right\rangle,
$$

to the dynamic spin structure factor $S_{\mathrm{k}}(\omega)$. In Eq. (13) $\omega$ cannot exceed $\left|E_{0}\right|$, following from the fact that we have ensured that $E_{0}$ is the eigenvalue with 
the largest magnitude.

For any finite system, $A_{\mathrm{k}}$ is a sum of $\delta$-functions and this can be replaced by a continuum starting at $\omega$ $=\Delta(\mathrm{k})$ for a large system. With $G_{\mathrm{k}}(\tau)$ or $Q_{\mathrm{k}}(t)$ computed using QMC calculations, the respective relations (13) or (14) can in principle be inverted using numerical analytic continuation. This procedure is very challenging, however, and it is not easy to extract the gap precisely with conventional methods such as the Maximum Entropy method [22], though one can extract the main dominant spectral features. To extract the lowest gap (and not the full spectral function), we model the excitations by just a small number of $\delta$-functions. With the precision of typical QMC data, $Q_{\mathrm{k}}(t)$ can be normally fitted very well with just a few $\delta$-functions (typically 3 to 5 ) over the full range of accessible times $t$. With this procedure, we expect the location $\omega_{1}$ of the lowest gap to accurately reproduce the lowest gap $\Delta(\mathrm{k})$, while the higher $\delta$-functions represent approximately the contributions of the continuum. In this fitting procedure, the extracted $\omega_{1}$ is to some extent affected by contributions of the higher states but does not change significantly when increasing the number of $\delta$ functions. We can therefore quite reliably extract the lowest gap, but not higher ones unless they are separated by significant subsequent gaps.

Given a set of $n \delta$-functions at energies $\omega_{i}$ with associated amplitudes $A_{i}$ normalized so that $\Sigma_{i} A_{i}=1$, one can compute the associated time dependent correlation function in analogy with Eq. (13) as

$$
Q_{\mathrm{k}}(t)=\sum_{i=1}^{n} A_{i}\left(1-\frac{\omega_{i}}{\left|E_{0}\right|}\right)^{t}
$$

Denoting the corresponding QMC-computed function by $\tilde{Q}_{\mathrm{k}}(t)$ and their statistical error by $\sigma_{t}$, the goodness of the fit is quantified in the standard way by $\chi^{2}$, based on a set of $N_{t}$ time points $\{t\}$ :

$$
\chi^{2}=\frac{1}{N_{t}} \sum_{\{t\}} \frac{1}{\sigma_{t}^{2}}\left(Q_{\mathrm{k}}(t)-\tilde{Q}_{\mathrm{k}}(t)\right)^{2} .
$$

where a uniform grid of time points with separation $N / 4$ operations was used up to a point $t_{\max }=N_{t}(N / 4)$ where the relative statistical error of $\tilde{Q}_{\mathrm{k}}(t)$ exceeds $5 \%$.
For the extracted gap to be reliable, the contribution of the lowest $\delta$-function to the fit must be significant at the longest times included. To monitor this long-time weight, we compute the relative contribution of the lowest $\delta$-function, denoted by $A_{1}(t)$, at the time $t_{\max }$ included in the fit:

$$
A_{1}\left(t_{\max }\right)=\frac{A_{1}\left(1-\omega_{1} /\left|E_{0}\right|\right)^{t_{\max }}}{\sum_{i=1}^{n} A_{i}\left(1-\omega_{1} /\left|E_{0}\right|\right)^{t_{\max }}} .
$$

This quantity should approach 1 for $t \rightarrow \infty$ if the lowest $\delta$-function is at the gap. It is close to 1 in all the fits that will be discussed below, indicating stable extraction of $\omega_{1}$.

The statistical error of the extracted gap is estimated using a bootstrap error analysis. With the underlying QMC data for the correlation function $C_{\mathrm{k}}(t)$ saved as $M$ bin averages (with typically $\mathrm{M} \sim 100$ 1000), bootstrap averages are constructed by selecting $M$ bins at random (i.e., allowing for the same bin to be selected multiple times). The above fitting procedures are then carried out repeatedly for a large number of these samples, and the standard deviation of the estimates is the statistical error of the gap in our procedure.

The fluctuations of the QMC data at different times are significantly correlated since these are measured in the same simulation. A statistically correct treatment of the data would require the inclusion of the full covariance matrix (instead of just its diagonal elements) in the definition of $\chi^{2}$. However, much of the covariance is already removed when the timecorrelations are normalized [by the denominator Eq. (11)], because the errors are correlated primarily by overall fluctuations in the normalization. Based on test cases, including ones included here, to obtain fully reliable results it is sufficient to use only the diagonal elements of the covariance matrix and define $\chi^{2}$. as in [17].

\section{Some Tests on The Heisenberg Spin Chain}

We here illustrate the gap extraction method described in the previous subsection using the example of the $S$ $=1 / 2$ Heisenberg spin chain with periodic boundary conditions, where spins interact with nearest neighbor exchange constant $J=1$;

$$
H=J \sum_{i=1}^{L} \mathbf{S}_{i} \mathbf{S}_{i}
$$



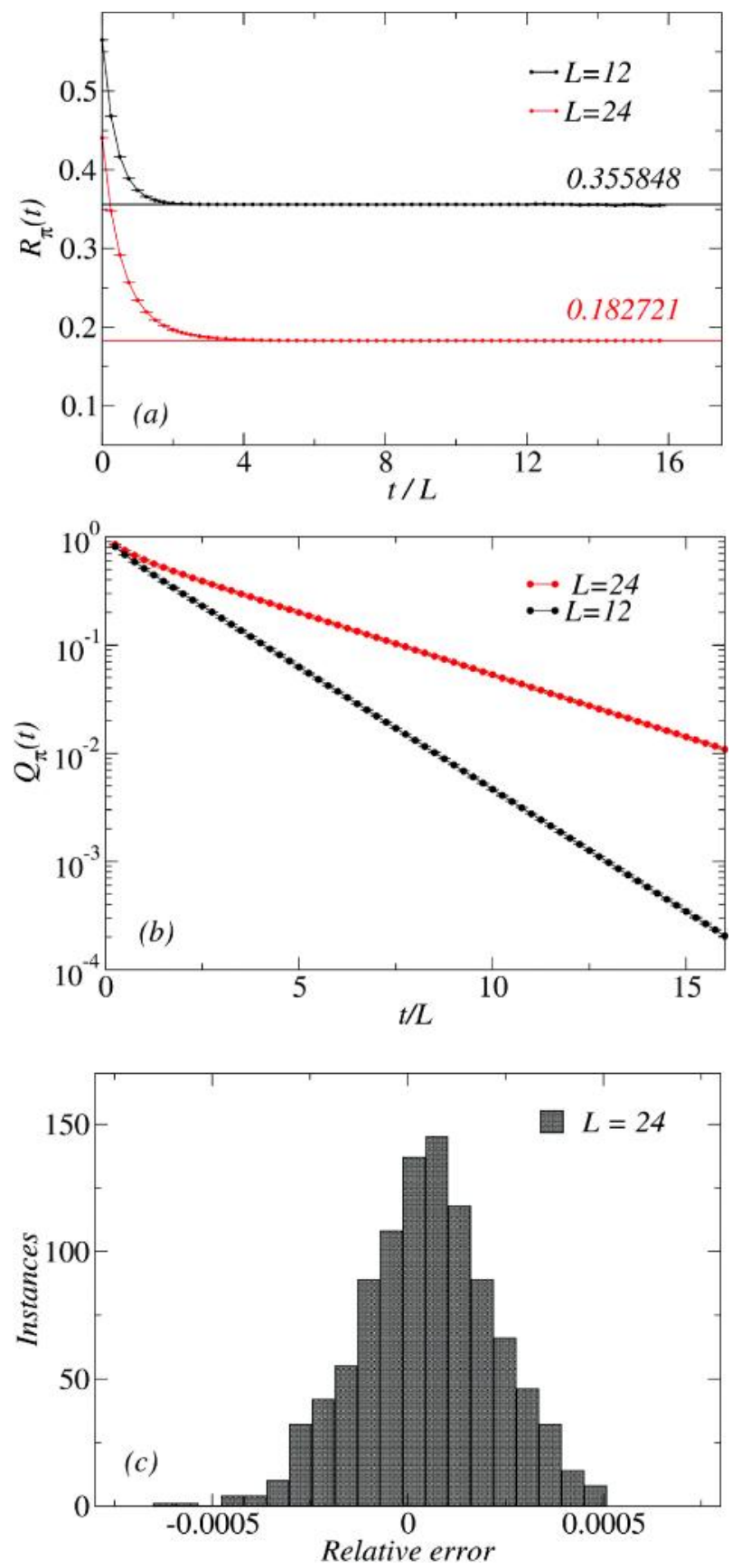

Fig. 2: (Fig. taken from Ref. [16]) (a) Correlation ratio $R_{\pi}(t)$, Eq. (12), shown for two Heisenberg chain sizes, $L=12$ and 24. The lines are the exact values of the triplet gap at this wave-vector (with the numerical values indicated as well). (b) The normalized correlation function $Q_{\pi}(t)$ for $L=12$ and $L=24$. (c) The distribution of the relative error of the gap $\Delta(\pi)$ extracted by fitting the data for $L=24$ in (b) using three $\delta$-functions. The histogram was generated from a large number of bootstrap samples of the QMC data. The relative error is calculated as $\left(\Delta-\Delta_{e}\right) / \Delta_{e}$ where $\Delta_{e}$ is the exact value of the gap. The exact value (corresponding to relative error 0 ) is seen to fall within a standard deviation of the distribution of the bootstrap samples
First, we compare the results of our numerics for the lowest triplet gap at $k=\pi$ for chain sizes $L=$ 12 and 24 with exact diagonalization (Lanczosmethod) results. As can be seen in Fig. 2(A), the quantity $R_{\pi}(t)$ defined in Eq. (12) indeed converges to the correct gap value in both the cases. Fig. 2(B) shows the normalized imaginary time correlation function $Q_{\pi}(t)$ for the two system sizes, and Fig. 2(C) shows the distribution of the gap error obtained using a bootstrap method for $L=24$, using a fit to three $\delta$ functions.

This analysis show that the gap obtained from the fit agrees statistically with the exact result, which here is within a standard deviation of the distribution obtained in the bootstrapping procedure, and the distribution itself closely matches a normal distribution. Thus, even with only three $\delta$-functions in the spectrum (which is clearly a much smaller number than what is contained in the full spectrum) we detect no systematic errors introduced by the fitted functional form, supporting the statement that the simplified description of the spectrum does not significantly affect the location of the lowest $\delta$-function (the gap). The number $n$ of $\delta$-functions that should be included in a given case depends on the statistical errors of the QMC data and the actual form of the spectral continuum. To determine $n$, we monitor $\chi^{2}$ as a

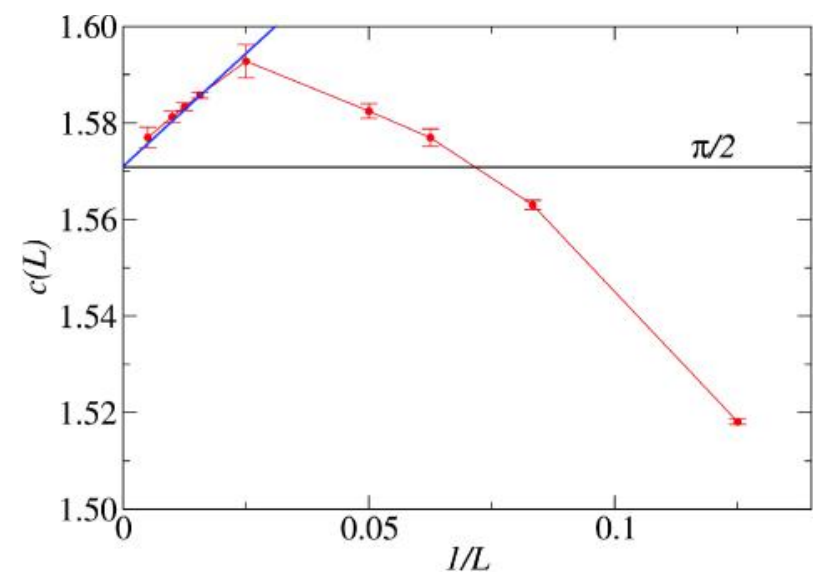

Fig. 3: (Fig. taken from Ref. 16) Velocity estimate for the Heisenberg chain from the triplet gap close to $k=0$; $c(L)=\Delta(k) / k$, where $k=2 \pi / L$, shown as a function of the inverse system size. The known velocity $c=\pi / 2$ in the thermodynamic limit is indicated by the horizontal line. The line through the larger system sizes is not a fit but is drawn to match the known velocity and the data for those system sizes 
function of increasing $n$ and stop when no improvements in the fit are observed.

For the Heisenberg spin chain, the spectrum of the triplet excitations is rigorously known based on the Bethe Ansatz solution [24]. The excitations consist of esssentially independent propagationg deconfined spinons carrying spin $S=1 / 2$. The "observable" singlet and triplet excitations are composed on an even number of such spinons. For momentum $k \rightarrow 0$ and $k$ $\rightarrow \pi$, the spectrum is linear with a known velocity of $c=\pi / 2$. The velocity of the spinons can be directly extracted from the lowest triplet gap at $k=2 \pi / L$ by using the simple estimator $L \Delta(2 \pi / L) /(2 \pi) \rightarrow c$ as $L$ $\rightarrow \infty$. Results for this quantity are shown in Fig 3 . We indeed observe the estimate approaching the correct value of $c$ as $L \rightarrow \infty$, but with a non-monotonic behavior with a maximum for $L \approx 40$ before an apparently linear asymptotic approach to the correct value. We note here that because of the nonmonotonic behavior seen in Fig. 3, a calculation using exact (numerical) diagonalization of the Hamiltonian would appear inconsistent with the known velocity, because large enough system sizes required to go beyond the maximum at $L \approx 40$ cannot be reached.

\section{The Velocity from Winding Number Fluctuations}

We here discuss how to use winding numbers to compute the velocity of propagating low-energy modes. This method has been known for some time, [25] and was recently applied to the 2D XY model [26] and the 2D Heisenberg model. [27] Even though the velocity estimator based on the excitation gaps discussed in the previous section is very direct and based on the fundamental definition of the velocity $c$ in terms of dispersion relation of the lowest energy excitations with respect to $\mathbf{k}$, this indirect method (for which a general proof is not present) is easier to apply and gives the correct velocity $\mathrm{c}$ for linearly propagating Goldstone and critical modes as we will numerically show here. We briefly recollect some key aspects about winding numbers in finite-temperature QMC simulations below before presenting results for the Heisenberg chain.

\section{Winding Numbers in QMC Simulations}

QMC simulations at finite temperature are based on some mapping of the partition function of a quantum system in $D$ dimensions to an effective $(D+1)$ - dimensional classical system, where the additional dimension of the configurations corresponds to imaginary (Euclidean) time. The effective length of the system in the time dimension is $c \beta$, where $\beta=1 /$ $T$ (setting $k_{B}=1$ ) and the configurations are timeperiodic. For a system with conserved particle number, which in the case of spin model corresponds to conserved magnetization along the quantization $(z)$ axis, imposing periodic boundary in the spatial dimensions leads to another, topological number associated with the configurations - the winding number representing permutations when particles are propagated once or multiple times around the periodic system. The winding numbers were first used in QMC calculations of the superfluid stiffness of interacting bosons [28].

The SSE QMC algorithm [15, 21] is based on a (high-temperature) Taylor expansion of the imaginarytime evolution operator (the Boltzmann operator) $e^{-\beta H}$. Each SSE configuration is associated with some power $H^{n}$ of the Hamiltonian propagating a basis state (here in the standard computational basis of $z$ spin components), and these powers are sampled stochastically to all contributing orders. The trace over all basis state is also sampled. The average expansion power $\langle n\rangle$ in this procedure is proportional to $\beta ;\langle n\rangle$ $=\beta\langle H\rangle \propto \beta N$. In SSE QMC simulations, the state propagation is broken up into individual paths corresponding to strings of $n$ of the individual local terms of the Hamiltonian, forming successions of $n$ evolving basis states, similar to those in path integrals. For a Heisenberg model the Hamiltonian terms are the diagonal operators $S_{i}^{+} S_{j}^{-}+S_{i}^{-} S_{j}^{+}$operators, the latter of which transport spin and are associated with currents $J_{a}= \pm 1$ in the lattice direction $a$ corresponding to the site-pair $i, j$. The winding number in the a lattice direction is defined in terms of the currents as

$$
W_{a}=\frac{1}{L_{a}} \sum_{p=1}^{n} J_{a}(p)
$$

where the index $p$ corresponds to the location of the transport "event" in the string of n operators and $L_{a}$ is the length of the lattice in the a direction. Defined in this way, the winding numbers are integers.

The spatial winding number $W_{r}$ measures the net spin transported around the periodic lattice in the $r$ direction in the course of the periodic propagation in 

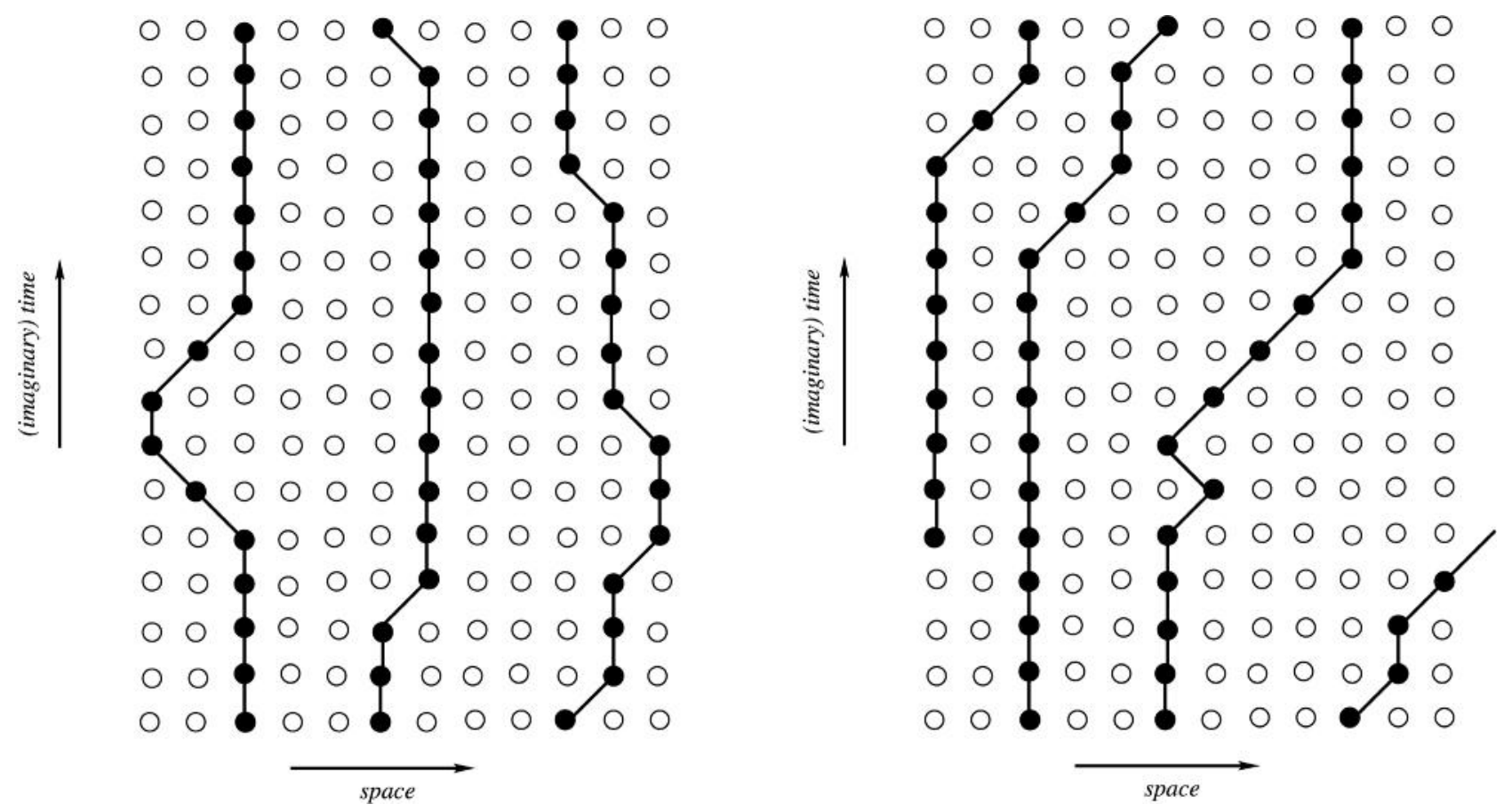

Fig. 4: Two cases of different spatial winding numbers displayed here for a one-dimensional $S=1 / 2$ system with 12 spins and periodic boundary conditions. The closed (open) circles represent spins with $S_{i}^{z}=+1 / 2(-1 / 2)$. (Left panel) The winding number here equals $W_{x}=0$ and no net spin is transported in imaginary time. (Right panel) The winding number here is non-zero and equals $W_{x}=+1$ (using Eq. 20) with a net spin being transported along the periodic $x$ direction during the propagation in imaginary time

imaginary time. A simple example of this is illustrated in Fig. 4 for 12 spins in a one-dimensional lattice with periodic boundary conditions in space, where the spin transport in imaginary time is caused by the offdiagonal $S_{i}^{+} S_{j}^{-}+S_{i}^{-} S_{j}^{+}$operators. Using Eq. 20, it is easy to verify that the left panel of Fig. 4 has $W_{x}=0$ while the right panel has $W_{x}=1$ with a net spin transport along the periodic space direction as the system is propagated in imaginary time. Since the total $z$ magnetization $M_{z}$ is conserved, one can also think of $M_{z}$ as a winding number; the net number of world lines crossing a plane drawn at an arbitrary time point perpendicular to the time axis, which is just the magnetization computed in the stored basis state;

$$
W_{\tau}=M_{z} \sum_{i=1}^{N} S_{i}^{z}
$$

The expectation values of the squared winding numbers are related to two important thermodynamic quantities; the spin stiffness,

$$
\rho_{s}=\frac{1}{2 \beta}\left(\left\langle W_{x}^{2}\right\rangle+\left\langle W_{y}^{2}\right\rangle\right),
$$

and the uniform magnetic susceptibility,

$$
\chi=\frac{\beta}{N}\left\langle M_{z}^{2}\right\rangle=\frac{\beta}{N}\left\langle W_{\tau}^{2}\right\rangle .
$$

The technicalities of implementing these observables in SSE calculations have been discussed extensively in the literature; see Ref. 15 for a recent review.

\section{The Cubic Criterion and the Velocity}

For a system with linearly dispersing low-energy modes with velocity $c$, the condition to reach a cubic regime in space-time for the effective $(D+1)$ dimensional classical system simulated in the finite- $T$ QMC algorithm corresponds to the effective length of the imaginary time dimension, $c \beta$, being equal to the linear dimension, $L$, of the system (shown schematically in Fig. 5). Here $c$ is a velocity that 
connects the engineering dimensions of space and time and should equal the velocity of the linearly propagating modes for a $z=1$ theory. The winding numbers characterize global fluctuations of the system in the different spatial and temporal directions. It is then natural to define a system as having cubic spacetime geometry (i.e., being equivalent in space and time) when the following relation

$$
\left\langle W_{r}^{2}\left(\beta^{*}\right)\right\rangle=\left\langle W_{\tau}^{2}\left(\beta^{*}\right)\right\rangle,
$$

holds at some unique value of $\beta=\beta^{*}(L)$ for given size $L$. The aspect ratio $L / \beta^{*}$ should then directly correspond to the velocity of the long-wave-length excitations when $L \rightarrow \infty$. In some cases this can be shown directly based on low-energy field theory [26, 27] of the Néel phase of quantum antiferromagnets but even in the absence of such descriptions, the arguments are very general (based on dimensional considerations) and one can expect the conclusion $c$ $=L / \beta^{*}$ to always hold for a system with linear dispersion, though we are not aware of any formal proofs in the general case. We now show below why a $\beta^{*}$ satisfying Eq. 24 always exists for the unfrustrated antiferromagnetic systems being considered here.

When $T \rightarrow \infty$, the magnetization fluctuations of any system are maximized and therefore $\left\langle W_{\tau}^{2}\right\rangle>0$ according to Eq. (23). For an unfrustrated antiferromagnet the ground state is a singlet with a singlet-triplet finite-size gap, $\left\langle W_{\tau}^{2}\right\rangle \rightarrow 0$ when $T \rightarrow 0$ for any finite system. In contrast to these limits of $\left\langle W_{\tau}^{2}\right\rangle$ for the spatial winding number in direction $r(r$ $=x, y, \ldots),\left\langle W_{r}^{2}\right\rangle \rightarrow 0$ when $T \rightarrow \infty$ on account of there being no quantum fluctuations when the imaginary-time length $\beta \rightarrow 0$ and there are no contributions from expansion powers $n>0$. In the limit $T \rightarrow 0$, for a system with long-range order (or a "quasi-ordered" 1D system with power-law decaying correlations), the stiffness constant converges to a non-zero value for any $L$, and according to Eq. (22) we must then have a divergence $\left\langle W_{r}^{2}\right\rangle \sim 1 / T$. These different behaviors of the spatial and temporal winding numbers versus temperature guarantees that there is a crossing point where Eq. 24 is satisfied at some unique value of $\beta=\beta^{*}(L)$ for given size $L$.

\section{Test on the Heisenberg Chain}

To find the point where the cubic criterion $\left\langle W_{x}^{2}\right\rangle=$ $\left\langle W_{\tau}^{2}\right\rangle$ is satisfied, a system is simulated at several values of $\beta$ in the region where $\left\langle W_{x}^{2}\right\rangle \approx\left\langle W_{\tau}^{2}\right\rangle$ based on initial explorations and knowledge of the approximate value of the velocity. We fit a low-order polynomial to the difference $\left\langle W_{x}^{2}\right\rangle-\left\langle W_{\tau}^{2}\right\rangle$ and solve the resulting equation for the $\beta$-value for which the

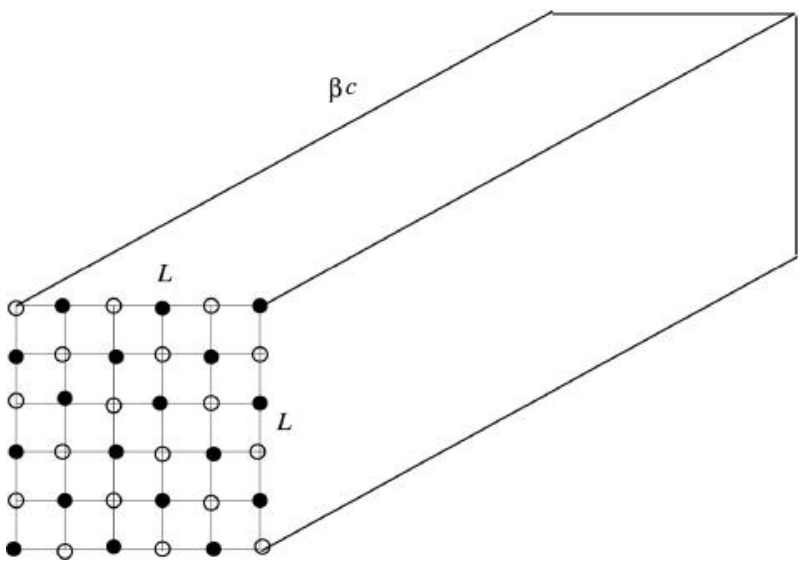

Fig. 5: The cubic regime in space-time for the effective (D + 1)-dimensional classical system simulated in a finite$T$ QMC algorithm corresponds to the effective imaginary time dimension, $c \beta$, being equal to the linear dimension $L$ of the system. Here $c$ relates the engineering dimensions of space and time

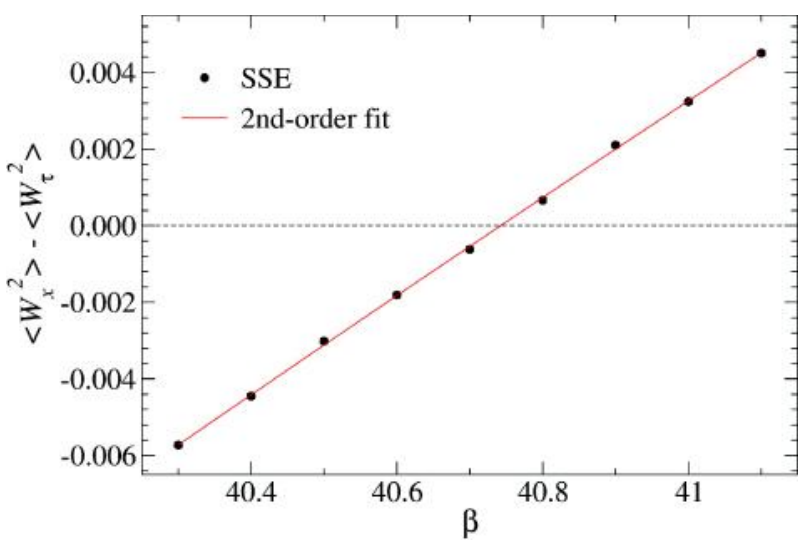

Fig. 6: (Fig. taken from Ref. 16) Difference between the spatial and temporal winding numbers versus the inverse temperature in simulations of a 64-site Heisenberg chain. A second-order polynomial has been fitted to the data points, and this curve is used to determine the value $\beta=\beta^{*}$ at which cubic condition $\left\langle W_{x}^{2}\right\rangle=\left\langle W_{\tau}^{2}\right\rangle$ holds (i.e., intersection with the horizontal dashed line) 


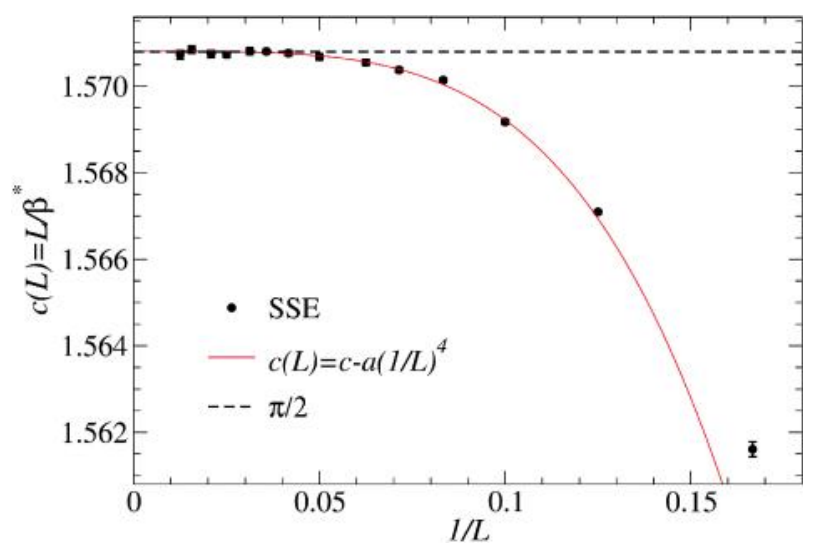

Fig. 7: (Fig. taken from Ref. 16) Size dependence of the velocity obtained with the cubic-criterion for the Heisenberg chain, along with a fit corresponding to a leading $\sim 1 / L^{4}$ size correction. The dashed line is at the rigorously known velocity $c=\pi / 2$

cubic criterion is satisfied. This procedure is illustrated in Fig. 6 for a Heisenberg chain with $L=64$ spins. From this procedure we obtain $c(L)=L / \beta^{*}(L)$, which can be extrapolated to $L \rightarrow \infty$.

Fig. 7 shows results of such a procedure for several chain lengths $L$, graphed versus $1 / L$. We are not aware of any theoretical predictions for the size dependence of this definition of $c(L)$, but the data for the larger systems are well described by a constant (the final infinite-size value of $c$ ) plus a term proportional to $1 / L^{4}$. Using this fitting form leads to a value of c completely consistent with the known value $c=\pi / 2$, as is clear from Fig. 7. Thus, the estimate based on winding number fluctuations gives the correct result even when the low-energy linearly propagating modes are spinons (fractionalized excitations with $S$ $=1 / 2$ ).

\section{D Heisenberg Model}

The 2D spin-1/2 Heisenberg model on the square lattice spontaneously breaks spin rotation symmetry at $T=0$ in the thermodynamic limit. This leads to gapless linearly dispersing Goldstone modes (spin waves) in the vicinity of the momenta $(0,0)$ and $(\pi$, $\pi$ ). The ground state in a finite periodic system is a total spin singlet. The long-range antiferromagnetic order in the thermodynamic limit is reflected in the energies of the $S>0$ low-energy excitations analogous to quantum rotor states [29] which collapse onto the ground state as $\Delta_{S} \sim 1 / L^{2}$ much faster than than the

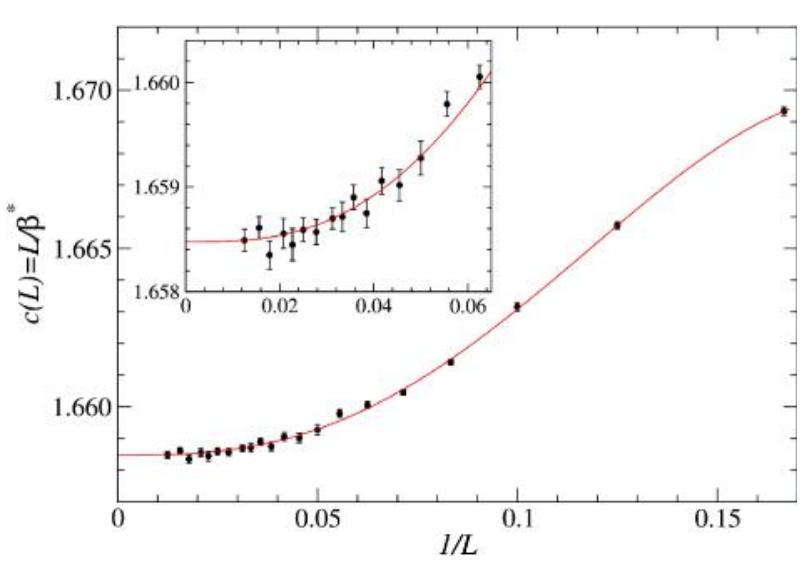

Fig. 8: (Fig. taken from Ref. 16) Size dependence of the velocity defined using the cubic-criterion for the $2 \mathrm{D}$ Heisenberg model, along with an fifth-order polynomial fit (including all system sizes $L \geq 6$ ) without linear and quadratic terms. The extrapolated velocity is $c=1.65847(4)$. The data for the largest system sizes are shown on a more detailed scale in the inset. The goodness of the $\chi^{2} /$ dof $\approx 0.8$.

spin wave excitations, $\Delta \sim 1 / L$. Such states, thus, become degenerate with the ground state as the system size increases, and have momenta $\mathbf{k}=(0,0)$ and $(\pi, \pi)$ for even and odd $\mathrm{S}$ respectively (such "rotor" states are absent at the other momenta). Superpositions of rotor states with $S$ up to $\sim L$ can then be formed which are ground states with fixed direction of the Néel vector (the staggered magnetization) which allows for symmetry breaking in the thermodynamic limit even though the staggered magnetization does not commute with the Hamiltonian. Such quantum rotor "tower of states" are in fact a generic finite-size signature of continuous symmetry breaking for many-body quantum systems [30] and are distinguished by the scaling of their gaps (from the ground state) that scales as $1 / L^{D}$ instead of $1 / L$ as is the case for the other gapless excitations, i.e., the Goldstone modes (here $L$ is the linear dimension of a $D$-dimensional system).

\section{Velocity from Winding Numbers}

Results based on the procedures discussed in the preceding section are shown in Fig. 8 versus the inverse system length. The figure shows the result of a polynomial fit and the extrapolated velocity with it is $c=1.65847$ (4). The above value of $c$ agrees within errors bars with the recent result using the same method by Jiang and Wiese, [27] but the error bar is 
almost an order of magnitude smaller. We note that in Ref. 27 no systematic extrapolation was carried out to the thermodynamic limit-instead an average was taken of results for system sizes in the range $L \in[24$, 64]. Looking at the data in Fig. 8 it is however clear that, with the small error bars on the SSE data achieved here, an extrapolation is necessary to obtain a result with no remaining finite-size effects. The above result [16] is the most precise spin-wave velocity reported to date for the 2D Heisenberg model. Spin-wave theory with corrections up to order $1 / S^{2}$ gave $c=1.6638(3)$, [31] where the uncertainty 3 in the last digit reflects estimated numerical errors from evaluations of challenging integrals. Thus, to this order, the spin-wave result deviates by only $0.3 \%$ from the correct value.

\section{Gap Scaling of Rotor States}

The quantum rotor excitation gap can be directly accessed by measuring the lowest triplet gap at $\mathbf{k}=$ $(\pi, \pi)$ which should scale as $L^{-2}$ instead of $L^{-1}$ for large $L$. This energy scale is related to the uniform (transverse) magnetic susceptibility $\chi_{\perp}$ as: [29]

$$
E(S, L)=\frac{S(S+1)}{2 L^{2} \chi_{\perp}}
$$

From the behavior of the lowest triplet gap up to system sizes $L=64$, as shown in Fig. 9, we indeed observe that $\Delta(\pi, \pi) \sim 1 / L^{2}$ at large $L$, but there are

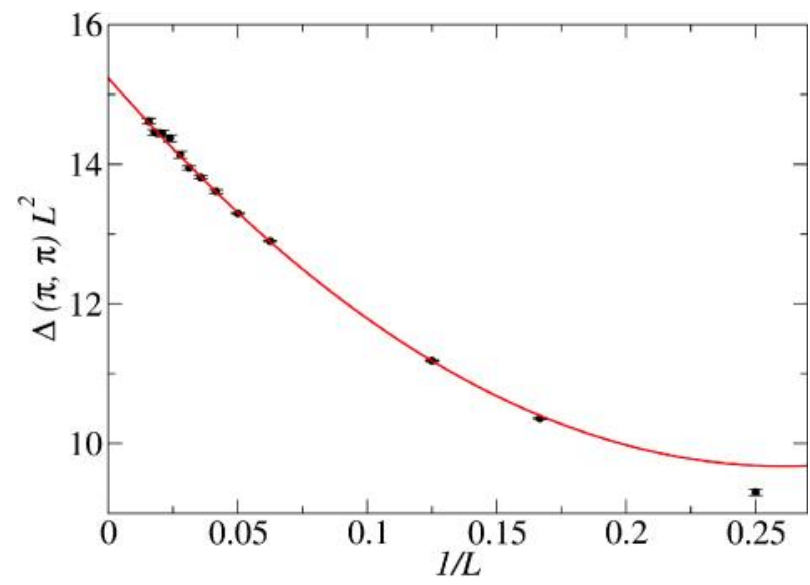

Fig. 9: (Fig. taken from Ref. 16) Size dependence of the lowest triplet gap $\Delta(\pi, \pi)$ multiplied by $L^{2}$ for the 2D Heisenberg model. This analysis shows that the gap scales as $1 / L^{2}$ for large $L$ and an extrapolation based on a polynomial in $1 / L$ gives the estimate $1 / 2 \chi_{\perp}=$ 7.62(2) for the uniform susceptibility also large corrections which we fit with additional higher-order powers of $1 / L$. The extrapolation to infinite size then gives $1 / 2 \chi_{\perp}=7.62(2)$. This is consistent with the value of susceptibility obtained using QMC calculations in small external magnetic fields to extract gaps between different spin sectors [32].

\section{Velocity from Gaps}

The velocity of the spin waves can be estimated directly from the triplet gaps in the vicinity of $(\pi, \pi)$ and $(0,0)$. We measure the triplet gap at $\mathbf{k}_{1}=(\pi+$ $2 \pi / L, \pi)$ [or, equivalently, at $(\pi, \pi+2 \pi / L)$, which we use for averaging] since the lowest triplet excitation energy is at $(\pi, \pi)$ and $\mathbf{k}_{1}$ is the closest allowed wavevector to $(\pi, \pi)$ for a periodic system with linear size $L$. We illustrate the gap extraction with both the ratio method and the simplified spectral function with fitted $\delta$-functions in Fig. 10, using the largest system sizes considered; $L=64$. Again, working with the spectral function produces much more stable results, though clearly the correlation ratio $R_{k}$ also converges to a constant consistent with the same gap.

Since in the thermodynamic limit, the excitation energy of the spin waves equals $E(\mathbf{k})=c|\mathbf{k}-(\pi, \pi)|$ in the vicinity of $(\pi, \pi)$, where $\mathrm{c}$ is the spin-wave velocity, the estimator

$$
c(L)=\frac{L}{2 \pi} \Delta(\pi+2 \pi / L, \pi)
$$

should converge to $c$ as $L \rightarrow \infty$. We graph this quantity in Fig. 11, and it converges to the value of $c$ obtained above from the winding-number method when $L \rightarrow$ $\infty$. Note that there is again a non-monotonicity as a function of $L$, similar to the case of the Heisenberg chain in Fig. 3.

\section{Bilayer Heisenberg Model}

We now consider the $S=1 / 2$ bilayer Heisenberg model which is a prototypical system to study quantum phase transitions in $2 \mathrm{D}$ and their equivalence to appropriate $(2+1) \mathrm{D}$ classical phase transitions [33$35]$. The quantum phase transition in the $2 \mathrm{D}$ bilayer is expected to be in the same universality class as the ?nite-temperature phase transition in the 3D classical Heisenberg ferromagnet and this has been confirmed using QMC simulations [36]. The Hamiltonian of the bilayer Heisenberg model is given by 

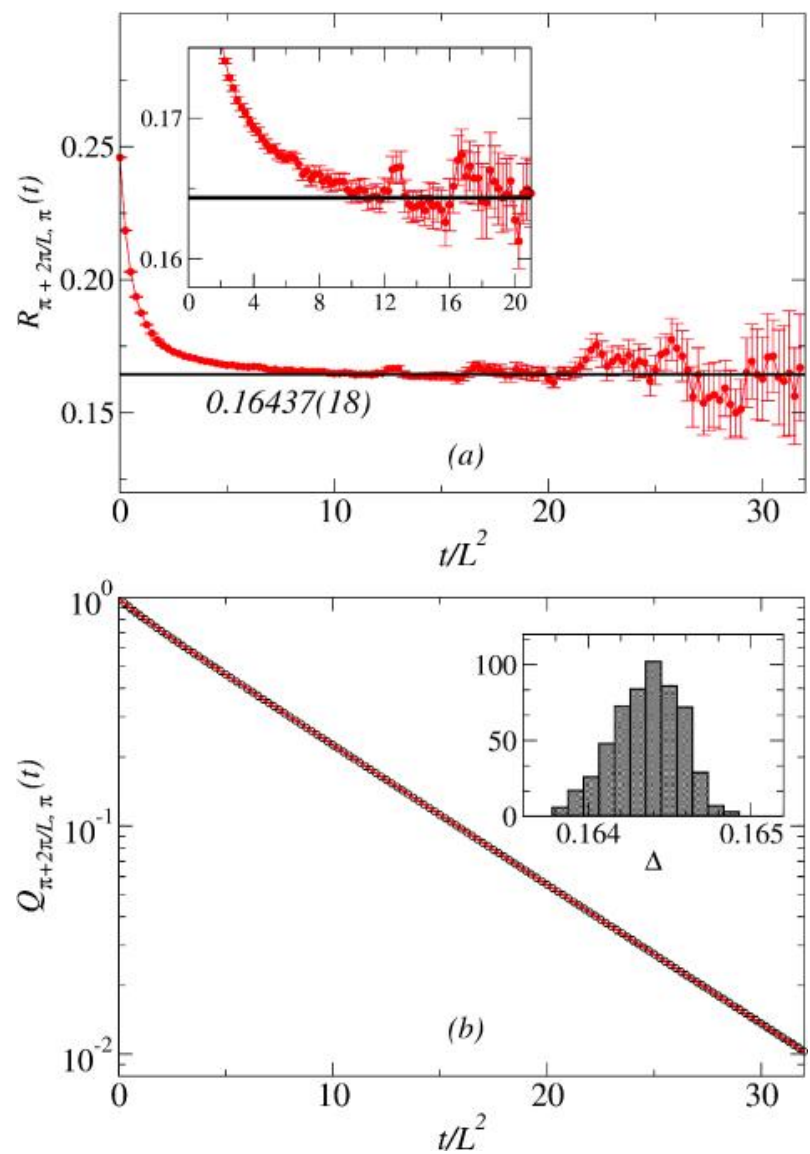

Fig. 10: (Fig. taken from Ref. 16) Extraction of the gap at $k=$ $(\pi+2 \pi / L, \pi)$ based on power-projected correlation functions, shown for the largest 2D Heisenberg system studied; $L=64$. (a) The ratio $R_{k}(t)$, Eq. (12) converges to a finite gap value but the error bars are large for long imaginary times. Fitting $Q_{k}(t)$ to four $\delta$ functions according to Eq. (13), as shown in (b), provides a more reliable gap estimation, as illustrated in the inset of (a). The thickness of the solid line here approximately represents the error bar. The inset of (b) shows the distribution of gap values obtained from a large number of different bootstrap samples of the QMC data, from which the error bar of the gap estimate was computed

$$
H=J_{1} \sum_{\langle i, j\rangle}\left(\mathbf{S}_{i, 1} \cdot \mathbf{S}_{j, 1}+\mathbf{S}_{i, 2} \cdot \mathbf{S}_{j, 2}\right)+J_{2} \sum_{i=1}^{L^{2}} \mathbf{S}_{i, 1} \cdot \mathbf{S}_{i, 2}
$$

where $\mathbf{S}_{i, a}$ represents a $S=1 / 2$ spin operator at site $\mathrm{i}$ of layer $a(a=1,2)$, and $\langle i, j\rangle$ denotes a pair of nearest neighbor sites on the square lattice of $L \mathrm{x} L$ sites with periodic boundary conditions. Both the couplings $J_{1}, J_{2}$ are positive (antiferromagnetic). As the ratio $g$ $=J_{2} / J_{1}$ is increased, there is a destruction of longrange Néel order at a critical $g_{c}$, beyond which the

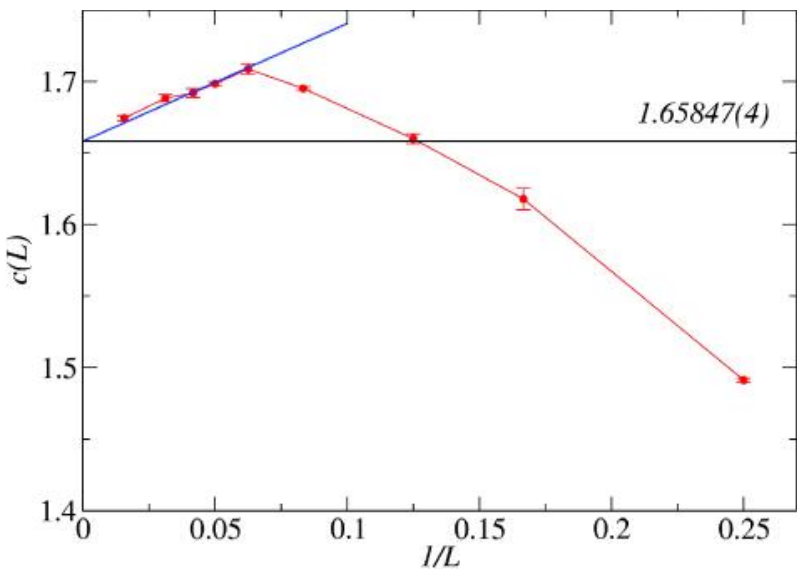

Fig. 11: (Fig. taken from Ref. 16) The spin-wave velocity estimator $c(L)$ defined in Eq. (26) shown for the 2D Heisenberg model as a function of $1 / L$. The velocity for large $L$ is consistent with the value obtained from the winding numbers (Fig. 8), shown as a horizontal line. The line through the values for the larger system sizes is a guide to the eye

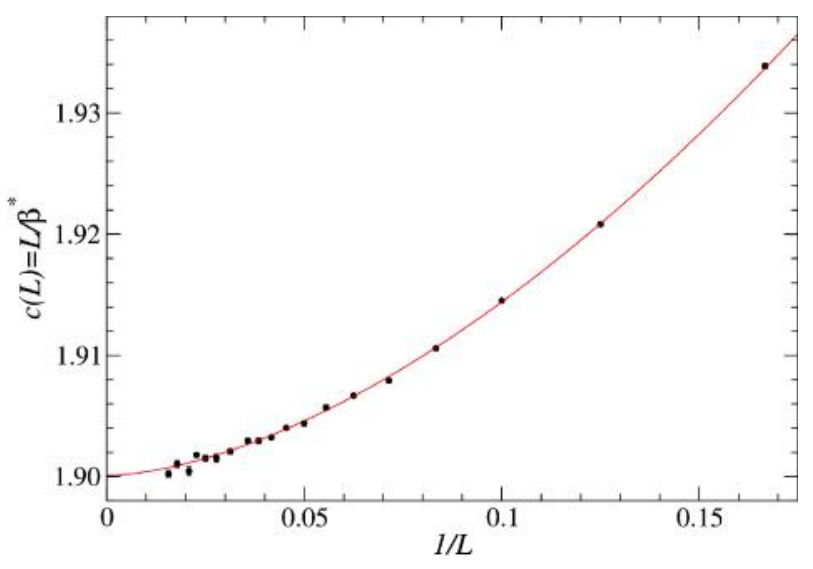

Fig. 12: (Fig. taken from Ref. 16) Size dependence of the velocity computed using the winding-number cubic criterion for the bilayer Heisenberg model at the estimated critical coupling ratio $g=\mathbf{2 . 5 2 2 0}$. The curve shows a quadratic fit of the form $c(L)=c+b / L^{a}$ to the $L \geq 8$ data, which gives the extrapolated velocity $c=$ $1.9001(2)$

system enters a quantum-disordered state with no broken symmetries. The best value available for the location of the critical point is $g_{c}=2.5220$ (1) [36] and we will use this value below.

\section{Velocity from Winding Numbers}

Results for the velocity based on the cubic windingnumber criterion at the critical point of the bilayer are 
shown in Fig. 12. A fit of the form $c(L)=c+b / L^{a}$ to the $L \geq 8$ data gives $c=1.9001$ (2). The value of $c$ is a few percent smaller than the spin-wave result [35] $c_{\mathrm{sw}}=1.96$ including $1 / S$ corrections at $g=2.51$ and $c_{\mathrm{sw}} \approx 2.03$ obtained [37] to order $1 / S^{2}$. A more sophisticated treatment beyond conventional spin-wave theory, including the effects of longitudinal fluctuations close to the critical point, gives $c=1.78$ when the expression $c=0.705 \times \mathrm{g}$ below Eq. (10) of Ref. 37 is evaluated with $g_{c}=2.522$. While these analytical values may appear reasonably close to (deviating by a few percent from) the presumably correct numerical value we have obtained here, the deviations are still much larger than in the case of the ordered 2D Heisenberg model, where the error of spin-wave result is only $0.3 \%$.

\section{Velocity from Gaps}

Since the bilayer has two sites per unit cell ( $a=1,2$ for each square-lattice point $i$ ), the triplet excitations have an extra label $k_{z}$ in $\mathbf{k}$ space which denote the inphase $\left(k_{z}=0\right)$ and out-of-phase $\left(k_{z}=\pi\right)$ spin excitations of the two layers, respectively. In the magnetically ordered Néel phase, the triplet excitations are gapless at $(0,0,0)$ and $(\pi, \pi, \pi)$, with the lowest excitation being at $(\pi, \pi, \pi)$ for a finite system. The spectrum is linear in the neighborhood of both the gapless points, which defines the corresponding spin wave velocity $c$. For a continuous phase transition, the spin wave velocity $c$ scales as

$$
c \propto\left(g-g_{c}\right)^{v(z-1)},
$$

where $z$ is the dynamical exponent and $\nu$ is the correlation length exponent. Thus, for $\mathrm{a} z=1$ transition as is the case for the Heisenberg bilayer at $g=g_{c}$, the velocity is regular at the critical point.

\section{Critical Point}

For measuring the velocity of the critical modes we first study the triplet gap at $(\pi+2 \pi / L, \pi, \pi)$. From the linearly of the spectrum at the critical point, we define the velocity estimator in analogy with the single-layer square lattice case (26) as

$$
c(L)=\Delta(\pi+2 \pi / L, \pi, \pi) L /(2 \pi)
$$

The behavior of this quantity as a function of $\mathrm{L}$ is shown in Fig. 13. Unlike the case of the Heisenberg chain (Fig. 3) and the single-layer (square lattice

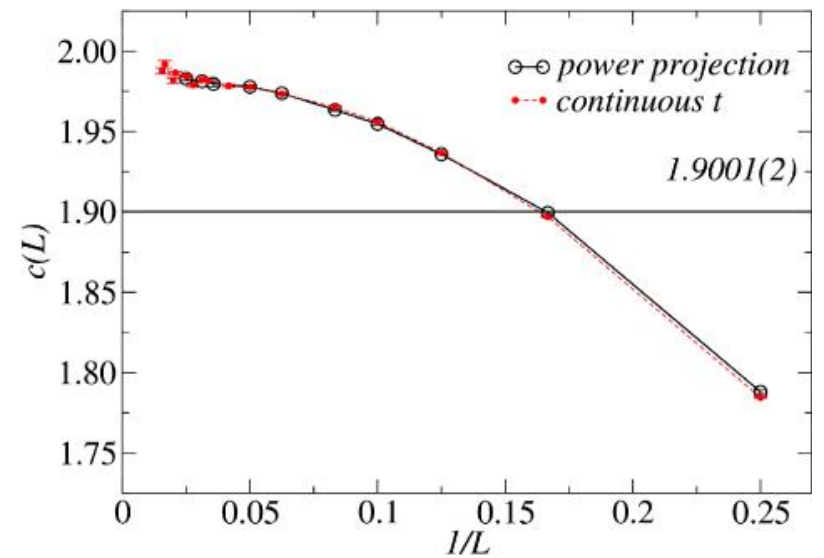

Fig. 13: (Fig. taken from Ref. 16) The velocity estimator (29) for the Heisenberg bilayer at its critical point shown as a function of $1 / L$. Results based on both powerprojection and exponential projection (continuous time) are shown and agree within error bars. The velocity estimate obtained from the winding numbers (Fig. 12) is shown as the horizontal line

antiferromagnet) (Fig. 11), the velocity estimate for the Heisenberg bilayer at criticality is notably higher than winding-numbers estimate, by about $5 \%$ as $L \rightarrow$ $\infty$. To extract the gap needed in Eq. (29) we have used both the power-projection QMC method with $\delta$ function fits to the correlation function and the generalized moment method applied to exponentialprojector QMC data, as detailed in Ref. 16 and Ref. 23. As seen in Fig. 13, the two methods give results that agree fully within statistical error.

At first sight, the potential reason for the disagreement with the winding-number result could be an over-estimation of the gap extracted from the correlation function at the critical point. Consider the full dynamic spin structure factor defined as

$$
S_{\mathbf{k}}(\omega)=\sum_{m}\left|\left\langle m\left|S^{z}(\mathrm{k})\right| 0\right\rangle\right|^{2} \delta\left(\omega+E_{0}-E_{m}\right) .
$$

In the Néel phase the structure factor has a $\delta$ function of weight $A_{d}(\mathbf{k})$ at an energy $\omega(\mathbf{k})$ which represents the magnon mode, as well as a continuum $A_{c}(\mathbf{k}, \omega)$ which does not extend below $\omega(\mathrm{k})$ and which decays rapidly to zero as $\omega \rightarrow \infty$ :

$$
S_{\mathbf{k}}(\omega)=A_{d}(\mathbf{k}) \delta(\omega-\omega(\mathbf{k}))+A_{c}(\mathbf{k}, \omega) .
$$

The velocity derived from $\omega(\mathbf{k})$ in the vicinity of $(\pi, \pi, \pi)$ is a regular function of $g-g_{c}$ and is by definition the correct velocity at the critical point. 
However, the weight in the $\delta$-function $A_{d}(\mathbf{k})$ (that represents the magnon mode) also smoothly goes to zero as the critical point is approached, with the spectrum evolving into purely a continuum reflecting the overdamped critical magnons. In our fitting scheme [see Eqs. (13) and (16)] used with the powerprojection QMC method, where the spectral function is represented by a small number of $\delta$ functions, $\omega(\mathbf{k})$ may be over-estimated, especially for large system sizes, when $A_{d}$ is very small close to the critical point. The velocity would then also be over-estimated, as it is in Fig. 13 (assuming that the winding-number result is correct). A similar distorting effect of the continuum may be expected also with the generalized moment method used with the exponential-projection QMC data. However, the $\delta$-function fits are stable with respect to the number of $\delta$-functions used, and the extrapolations used with the generalized moment method are also stable. The perfect match between the two methods within their statistical errors also shows that the gaps are determined correctly and the reason for the disagreement with the winding-number method must be sought elsewhere.

To search for a possible flaw in the velocity estimation based on Eq. (29), we next analyze the details of the dispersion relation around $(\pi, \pi, \pi)$, using gaps extracted with the generalized moment method. The dispersion should be an asymptotically linear

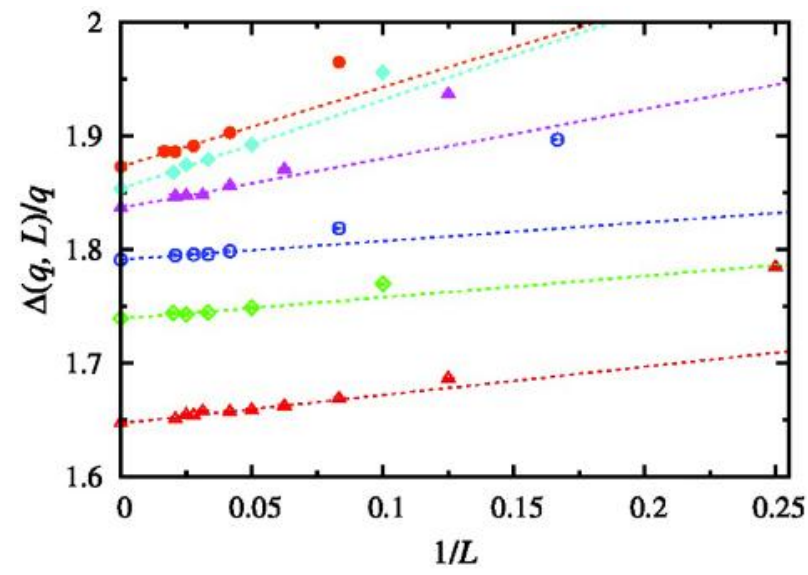

Fig. 14: (Fig. taken from Ref. 16) Momentum dependent gap, with $q$ defined relative to $(\pi, \pi, \pi)$, divided by the respective $q$. The data are shown versus $1 / L$ for $q=$ $2 \pi / 4$ (open triangles), $2 \pi / 5$ (open diamonds), $2 \pi / 6$ (open circles), $2 \pi / 8$ (solid triangles), $2 \pi / 10$ (solid diamonds), $2 \pi / 12$ (solid circles). Linear fits in $1 / L$ are shown for each momentum, with only data for sufficiently large $L$ included for each $q$

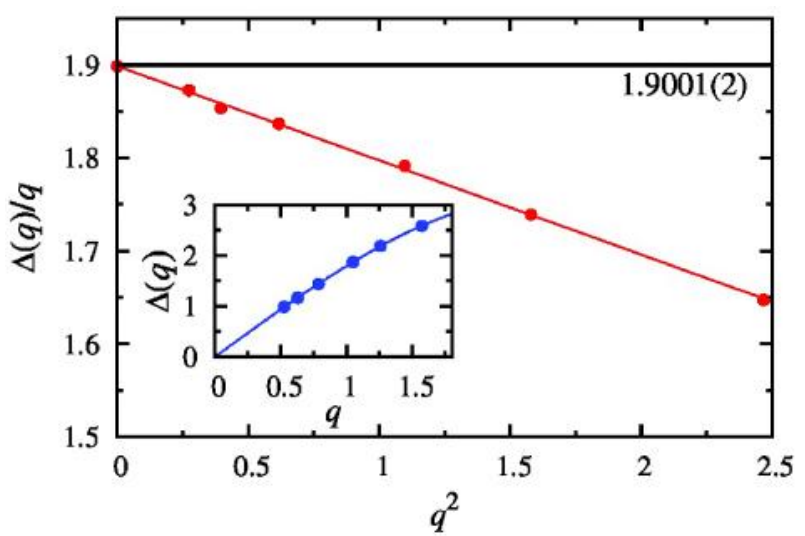

Fig. 15: (Fig. taken from Ref. 16) Extrapolation of the critical bilayer velocity using the gaps for $q \rightarrow 0$. The infinite-size velocity is estimated as the limiting value $c=1.899(2)$, which is consistent with the estimation from the winding numbers, $c=1.9001(2)$, indicated by the horizontal line. A linear function in $1 / q^{2}$ is used for the extrapolation. The inset shows the calculated dispersion

function of the wave number, $\Delta(\mathbf{q})=c q$, where $\mathbf{q}$ is the momentum relative to the gapless point $(\pi, \pi, \pi)$;

$$
\mathbf{q}=\mathbf{k}-(\pi, \pi, \pi) .
$$

We should then have $\lim _{q \rightarrow 0} \lim _{L \rightarrow \infty} \Delta(\pi, L) / q$ $=c$. There could potentially be an issue with the order of the limits $q \rightarrow 0$ and $L \rightarrow \infty$, which with Eq. (29) are taken simultaneously as we use $q=2 \pi / L$.

To investigate the formally correct limit of taking $L \rightarrow \infty$ first and then $q \rightarrow 0$, the gaps are analysed at fixed $q$ and varying $L$, to find the corresponding gap values as a function of the momentum in the thermodynamic limit. The $L$ dependence of the gaps at several different $q$-values are shown in Fig. 14. We observe that the size correction to the gap for sufficiently large $L$ is linear in $1 / L$ and therefore extrapolate the data to infinite size using simple line fits. Using these gaps $\Delta(q)$, we finally extrapolate the velocity as $c=\lim _{k \rightarrow 0} \Delta(q) / q$, as shown in Fig 15 . The behavior is fully consistent with a linear dispersion in the limit $q \rightarrow 0$, and empirically we find that the leading correction is of order $q^{2}$. The final result for the velocity extracted from these gaps is $c=1.899(2)$, which is fully consistent with the result based on the winding numbers. Thus, the reason for the previous disagreement is indeed that Eq. (29) does not represent the correct order of limits, and there are no flaws in the extraction of the gaps. 
This more detailed analysis also shows the reason for the over-estimation of the velocity based on Eq. (29). As is clear from Fig 14, the gaps converge to their infinite size values as $1 / L$ for sufficiently large $L$. In fact, we see that the gaps at fixed momentum $k$, where $\mathrm{k}$ is close to but not equal to $(\pi, \pi, \pi)$, follows the following behaviour for large $L$ :

$$
\begin{aligned}
& \Delta(q, L)=\Delta(q, L \rightarrow \infty)+\frac{A(q)}{L^{2}}(\text { gapless }) \\
& \Delta(q, L)=\Delta(q, L \rightarrow \infty)+\frac{B(q)}{L}(\text { critical }) \\
& \Delta(q, L)=\Delta(q, L \rightarrow \infty)+a(q) \mathrm{e}^{-b(q) L} \text { (gapped) }
\end{aligned}
$$

In the gapless and the gapped phases, the size correction decays sufficiently rapidly, so that $\Delta(q=$ $2 \pi / L, L \rightarrow \infty) L /(2 \pi)=c$. However, at the critical point, the estimator $\Delta(q, L) L / 2 \pi$ instead converges to $c+B(q \rightarrow 0) / 2 \pi$.

\section{Quantum Paramagnetic Phase}

For completeness we next extract the velocity of the propagating triplet excitations, or triplons, in the quantum-disordered phase of the bilayer. We choose two points well away from $g=g_{c}$, at $g=3$ and $g=4$. The lowest triplet gap at $(\pi, \pi, \pi)$ now converges to a finite ( $g$ dependent) value as $L \rightarrow \infty$, because the quantum paramagnetic phase is gapped. In the vicinity of $(\pi, \pi, \pi)$, the spectrum is expected to behave generically as

$$
\Delta(q)=\sqrt{\Delta_{0}^{2}+c^{2} q^{2}} \approx \Delta_{0}+\frac{c^{2} q^{2}}{2 \Delta_{0}},
$$

where $\Delta_{0}$ denotes the triplet gap at wavevector ( $\pi$, $\pi, \pi), c$ is the velocity of the gapped triplons, and $q$ is again measured relative to $(\pi, \pi, \pi)$. We use both the approaches (ways of taking $q \rightarrow 0$ and $L \rightarrow \infty$ ) discussed above to estimate the triplon velocity $\mathrm{c}$ and they give consistent results in the gapped phase; see Fig. 16 for the analysis at $g=3$. We obtain $c=$ 1.973(4) and $c=2.159(6)$ for $g=3$ and $g=4$, respectively.

The simple estimator (29) should give the correct velocity in the gapped phase, as discussed above, and we further check for consistency of the approach by also analyzing the gap at $q=4 \pi / L$, in addition to the smallest momentum $q=2 \pi / L$ (both extrapolations shown in Fig. 16, upper panel). The same velocity is also obtained using these extrapolated gap values representing the limit $L \rightarrow \infty$ before $q \rightarrow 0$, as shown in Fig. 16, bottom panel.

\section{Level Spectroscopy of $J-Q$ Model}

A central concept in the theory of continuous phase transitions (both thermal and quantum) is that of the order parameter. According to the accepted LandauGinzburg-Wilson (LGW) paradigm, [13, 38, 39] near a continuous phase tranition, the long distance properties of the system can be described by a continuum field theory written in terms of the order parameter field(s) and its gradients, where all terms consistent with the symmetries of the order parameter(s) are allowed. Are there phase transitions that lie outside this LGW paradigm? Following intriguing numerical results which suggested violations of LGW predictions [40, 41] the deconfined quantum critical (DQC) point was proposed as a scenario beyond the standard paradigm, [42, 43]. Here the critical theory is most naturally described in terms of fractionalized degrees of freedom that emerge at long distances, and not in terms of the order parameter fields. The order parameter fields characterizing the conventional orders on either side of the critical point emerge as composites of these fractionalized fields. These fractional objects should have prominent signatures in excitation spectra and spectral functions in the neighborhood of such critical points and should also control the finite-temperature physics in the quantum-critical fan around a $T=0$ DQC point. Below, we discuss results obtained in Ref. [17] for the low-energy excitations at the DQC point of a 2D quantum magnet, the $J-Q$ model.

The DQC point considered here separates states with Néel order and VBC order, [44] realized with the $J-Q$ spin-1/2 Hamiltonian, [45]

$$
H=-J \sum_{\langle i j\rangle} P_{i j}-Q \sum_{\langle i j k l\rangle} P_{i j} P_{k l},
$$

where $P_{i j}=1 / 4-\mathbf{S}_{i} \cdot \mathbf{S}_{j}$ is a singlet projector on sites $i j$, $\langle i j\rangle$ denotes nearest-neighbor sites (links) on a periodic square lattice with $L^{2}$ sites, and $\langle i j k l\rangle$ denotes a pair of links on a $2 \times 2$ site plaquette. The summations are over all links and plaquettes; thus $H$ maintains all 

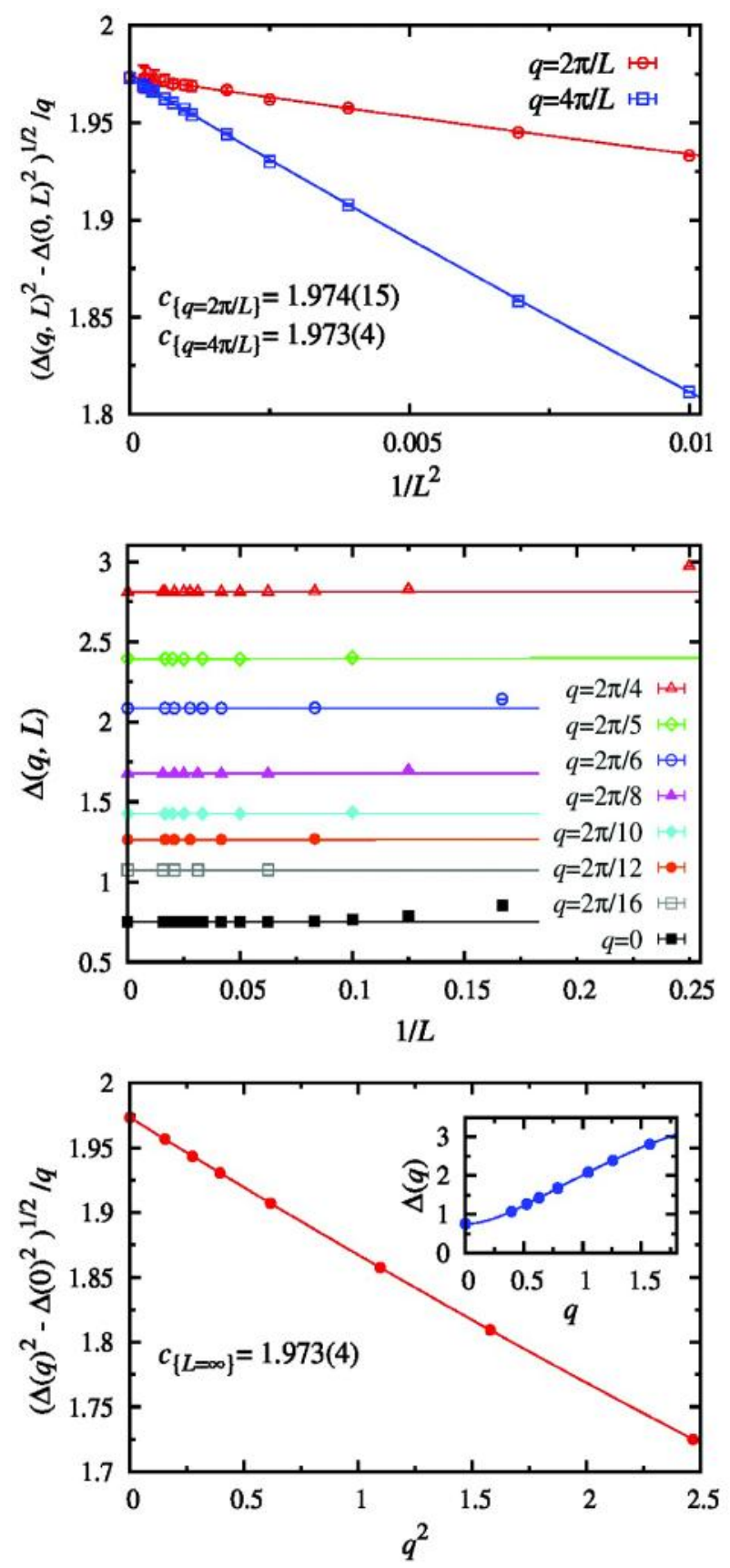

FIG. 16: (Fig. taken from Ref. 16) Velocity extraction for the bilayer at $g=3$ (quantum paramagnetic phase). The top panel shows the size convergence of velocity estimates based on two momenta close to the antiferromagnetic momentum ( $q$ being the deviation from this momentum). The middle panel shows the system-size dependence of the gaps at several values of $q$. The bottom panel shows the infinite-size extrapolated velocity defined versus $q$, obtained from the fits in the middle panel and graphed versus $q^{2}$. The $q \rightarrow 0$ extrapolation by a polynomial (red curve) agrees with the different order of the limits taken in the top panel. The inset shows the dispersion relation

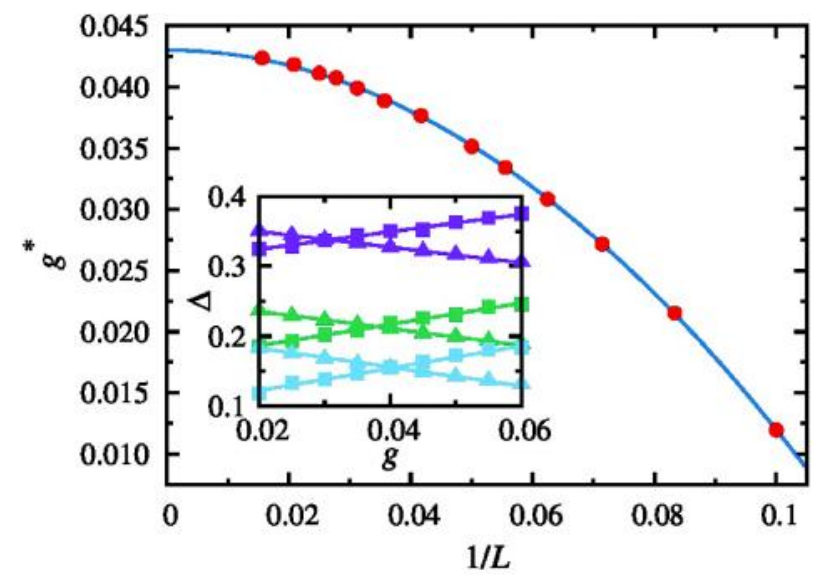

Fig. 17: (Fig. taken from Ref. 17) Extrapolation of the transition point from the coupling $g *(L)$ at the crossing between the lowest triplet and singlet gaps. A power-law fit, $g^{*}(L)-g_{c} \propto L^{-\sigma}$, for $10 \leq L \leq 64$ gives $g_{c}=0.04301(8)$ and $\sigma=2.00(1)$ (with $\chi^{2} / N_{\text {dof }} \approx 1.4$ ). The inset shows triplet (triangles) and singlet (squares) gaps for $L=16,24$, and 32 (top to bottom)

the symmetries of the square lattice. The $Q=0$ case is the standard antiferromagnetically-ordered Heisenberg model, and when $Q / J$ is sufficiently large, $Q / J \gtrsim 22$, projection of correlated singlets leads to spontaneous columnar dimerization and loss of antiferromagnetic order. In contrast to frustrated Heisenberg systems, like the $J_{1}-J_{2}$ model, that may also harbor VBC states and DQC points [46-48], the $J-Q$ model is not affected by sign problems and can be studied using QMC simulations on large lattices [15].

The existence of the DQC point has been addressed in numerous studies of the $J-Q$ model, [ 45 , 49-55]. Unusual scaling behaviors observed in these studies can now be accounted for by a scaling hypothesis incorporating the two divergent length scales of the theory, a standard correlation length and a scale related to emergent $U(1)$ symmetry of the VBC fluctuations [56]. While there are still important unsettled questions remaining, e.g., on the fundamental origins of the anomalous scaling [56] and an apparent emergent $\mathrm{SO}(5)$ symmetry [57], there is now little doubt that the transition is continuous (instead of weakly first order, as had been claimed in some studies [50, 58, 59]).

\section{Level Spectroscopy}

We first use level spectroscopy to locate the transition 
point and extract the critical gap scaling exponent. Different types of ground states are associated with different low-energy excitations, which can lead to crossings of energy levels with different quantum numbers as a function of the control parameter that drives the quantum phase transition. The finite-size scaling of the crossing point provides a remarkably good estimate of the critical point in several 1D systems, [60-62]. However, this method had not been used in $2 \mathrm{D}$ because exact diagonalization can only access a very limited range of linear dimension $L$. As for the $2 \mathrm{D} J-Q$ model, the triplet excitation is gapless in the Néel phase (the lowest triplet being a quantum rotor state with gap scaling as $1 / L^{2}$ [63]), while it is gapped in the VBC phase. In contrast, the lowest singlet gap of a finite system decreases exponentially with the system size in the VBC phase, while it converges to a finite value in the Néel phase. Therefore, the lowest triplet $(S=1)$, which is at $k=$ $(\pi, \pi)$, and singlet $(S=0)$, at $k=(\pi, 0)$ and $(0, \pi)$, cross each other at a coupling which converges to the transition point in the thermodynamic limit.

Figure 17 presents results, [17] with examples of level crossings shown in the inset and the finitesize drift of the crossing points $g^{*}(L)$ analyzed in the main figure. The crossing points have been fitted to a constant (the infinite-size critical point) with a powerlaw correction $\propto L^{-\sigma}$, with $\sigma=2.00(1)$. The critical point $g_{c}=g^{*}(L \rightarrow \infty)=0.04301(8)$, or $(J / Q)_{c}=$ $0.04494(9)$, is in reasonably good agreement with a recent, more precise estimate $(J / Q)=0.04468(4)$ [56]. The data for the gap at the crossing point is shown in

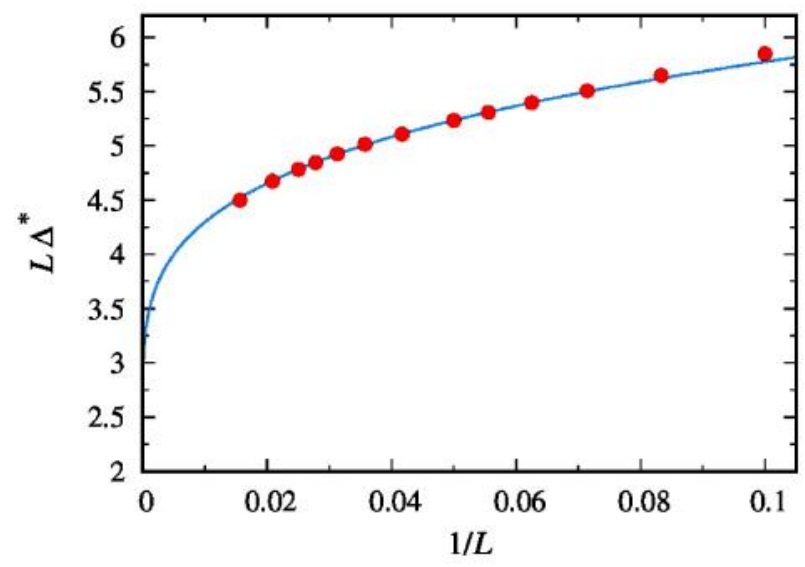

Fig. 18: (Fig. taken from Ref. 17) Scaling of the gap at the crossing point. A fit to the form $L \Delta^{*}=a+b L^{-\tau}$ for 16 $\leq L \leq 64$ gives $\pi=0.26(4)\left(\right.$ with $\left.\chi^{2} / N_{\text {dof }} \approx 1.8\right)$

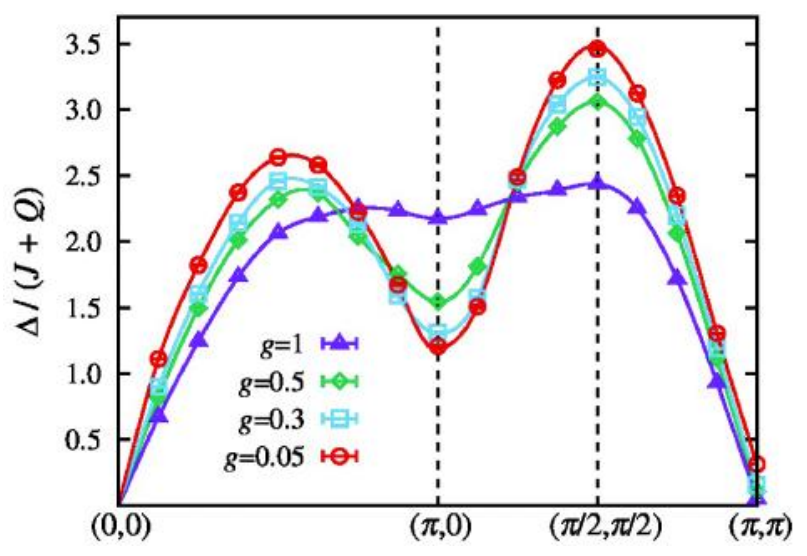

Fig. 19: (Fig. taken from Ref. 17). Dispersion relation of the lowest triplet for $L=16$ systems at several coupling parameters. The splines connecting points are only guides to the eye

Fig. 18. Given that the expected dynamic exponent $z$ $=1$, we here show the crossing gap $\Delta=\Delta_{s}=\Delta_{t}$ multiplied by the system size $L$, and again fit with a power-law correction; $\propto L^{-\tau}$ with $\tau=0.26(4)$.

\section{Linear Dispersion of Spinons}

We next discuss results for the dispersion curve around the DQC point and investigate carefully the finite-size scaling of the lowest gaps. We will see that at the critical point, there are both gapless singlets and triplets at $\mathbf{k}=(0,0),(\pi, 0),(0, \pi)$, and $(\pi, \pi)$. [17] In addition, the velocities around the gapless modes have been studied by the winding-number method and the direct-gap measurement [17] and it was found that a unique velocity appears around the multiple gapless modes. These findings strongly indicate that the low-energy excitation is formed by linearly dispersing spinons.

\section{Full Dispersion}

We study the dispersion of the lowest triplet. Figure 19 shows results for $L=16$ and several values of $g$ along a standard path in the Brillouin zone. Spin-wave theory for the Heisenberg model produces a magnonexcitation energy that is in generally good agreement with numerical calculations [63]. The main discrepancy is at the antiferromagnetic zone boundary, the line from $k=(\pi / 2, \pi / 2)$ to $(\pi, 0)$, where in spin wave theory to order $1 / \mathrm{S}$ there is no dispersion. Numerical calculations show a $10 \%$ lower energy at $(\pi, 0)[64,65]$. The minimum at $(\pi, 0)$ has been termed 
the "roton minimum" [66], in analogy with the local dispersion minimum in ${ }^{4} \mathrm{He}$. It has been argued that it originates from interactions between the transverse (magnon) and longitudinal ("Higgs") modes. In the $J$ $Q$ model, we can see that the differences between $k$ $=(\pi / 2, \pi / 2)$ and $(\pi, 0)$ increase dramatically as the critical point is approached. The reduction in $(\pi, 0)$ energy is in accord with a variational argument [67], according to which the triplet gap at $(\pi, 0)$ must vanish if the Néel-to-VBC transition is continuous. For the relatively small system in Fig. 19, the expected gapless $(\pi, 0)$ mode is not yet apparent and requires a finitesize analysis close to $g$, as we discuss later. Even with the data in Fig. 19, it is now clear that the weak roton minimum of the Heisenberg model is due to VBC fluctuations (which should also be related to emergent gauge bosons [68]), as also discussed in [69], which are strengthened as $Q / J$ is increased and push the minimum down to 0 as the DQC point is approached.

\section{Finite-size Scaling of Gaps}

In addition to the gapless triplet at $\mathbf{k}=(\pi, \pi)$ and singlets at $(\pi, 0),(0, \pi)$, we also expect a gapless triplet at $\mathbf{k}=(0,0)$, as is well known in the Heisenberg model and which is also reflected in Fig. 19. Next we will see that actually there are both gapless singlets and triplets at all these four points, $\mathbf{k}=(0,0),(\pi, 0)$, $(0, \pi)$, and $(\pi, \pi)$; that is, there are eight gapless excitation modes in total. We focused the calculations

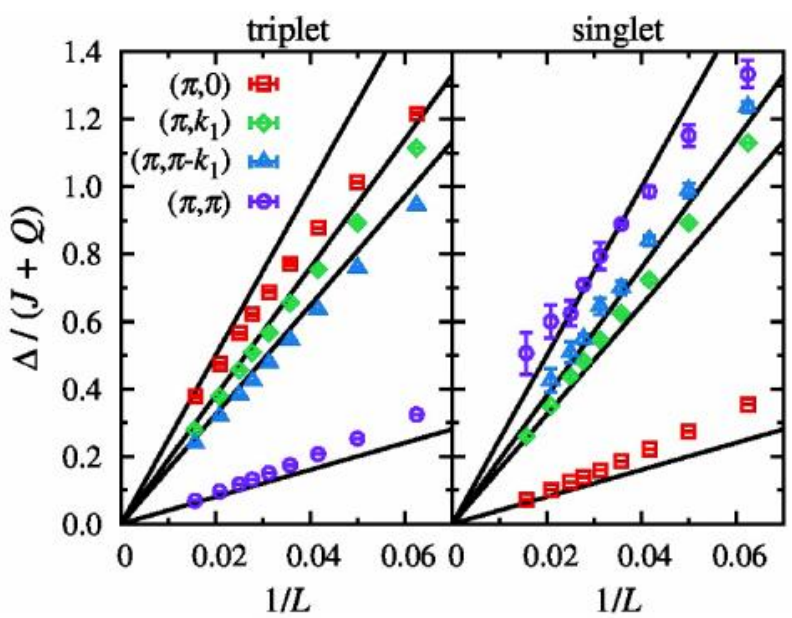

Fig. 20: (Fig. taken from Ref. 17) Triplet (left) and singlet (right) gaps at several wave vectors for systems close to the $D Q C$ point $(J / Q=\mathbf{0 . 0 4 5})$. The smallest wavevector increment $2 \pi / L$ is denoted by $k_{1}$. The solid lines illustrate the expected critical form $\Delta \propto L^{-1}$ with $\Delta_{t,(\pi, q)}=\Delta_{s,(\pi, \pi-q)}$ imposed at $J / Q=0.045$, close to the estimated critical value, and extracted the gaps $\Delta_{\mu, \mathbf{k}}$ at several wave vectors. Results are displayed in Fig. 20, along with fits to the expected $1 / L$ forms for $z=1$ criticality. A very interesting observation is a singlet-triplet symmetrya generalization of the equivalence of the lowest $s, t$ gaps: $\Delta_{t,(\pi, q)} \approx \Delta_{s,(\pi, \pi-q)}$ is seen for $q=0,2 \pi / L, \pi-$ $2 \pi / L$, and $\pi$. The singlets are a bit higher than the corresponding triplets, likely because of higher-order irrelevant fields as the differences appear to vanish as $L \rightarrow \infty$. In Fig. 20 we draw lines with the same prefactors in $1 / \mathrm{L}$ for the corresponding gaps. These findings strongly suggest that the system has gapless singlet and triplet excitations at $(0,0),(\pi, 0),(0, \pi)$, and $(\pi, \pi)$, with a remarkable connection between the finite-size corrections for singlets and triplets that may again be related to emergent $\mathrm{SO}(5)$ symmetry.

\section{Velocity of Propagationg Modes}

We present an estimate of the velocity obtained using winding numbers first, and thereafter discuss the more direct approach using gaps. In Ref. [16] several highprecision tests for both ordered and critical systems were presented. We have used the winding-number method also for the $J-Q$ model and present results for several system sizes at $J / Q=0.045$ in Fig. 21 .

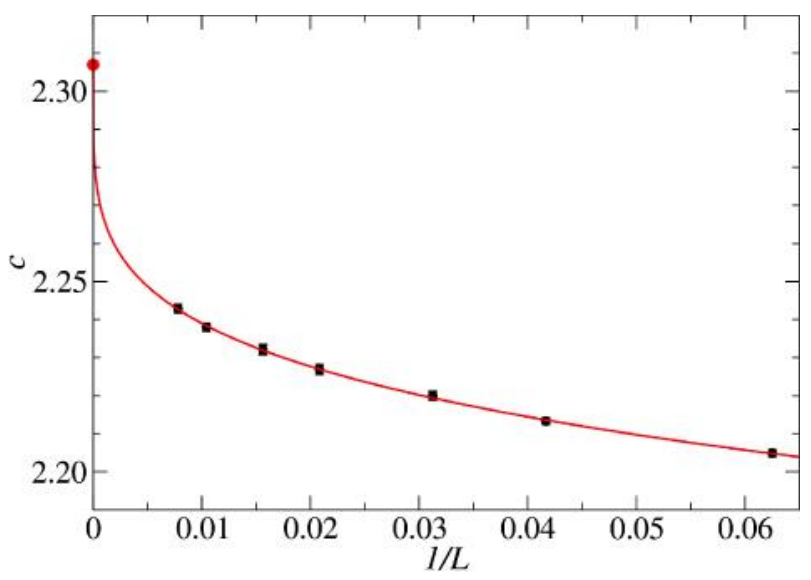

Fig. 21: (Fig. taken from Ref. 17). Velocity estimates for the $J-Q$ model at $J / Q=0.045$ [close to its critical point, $(J / Q) \mathrm{c} \approx 0.447]$ extracted using the cubic criterion Eq. (24). The unit of c corresponds to setting the lattice constant to 1 and the energy scale $J+Q=1$. The curve shows a fit including a power-law correction $\propto L^{-\omega}$ to the infinite-size velocity. The fit with error analysis gives $c=2.31(5)$ and $\omega=0.24(8)$ 
The finite-size data are in excellent agreement with a constant plus a finite-size correction $\propto L^{-\tau}$, with the velocity $c /(J+Q)=2.31(5)$ in the thermodynamic limit and $\omega=0.24(8)$. As shown in Fig. 22, the value of $\mathrm{c}$ is in excellent agreement with the velocity extracted using energy gaps, and it is also in good agreement with a previous QMC calculation (where, however, no scaling correction was used in the analysis) [25].

We next extract the velocity $c$ of excitations, using $\mathbf{k}$ points away from the gapless points $K_{0}$ by amounts $k_{1}, k_{2}$, where $k_{n}=n 2 \pi / L$ :

$$
c_{\mu, \mathbf{K}_{0}}(L) \equiv(L / 2 \pi)\left[\Delta_{\mu, \mathbf{K}_{0}}(L)-\Delta_{\mu, \mathbf{K}_{0}+\mathbf{K}_{1}}(L)\right] .
$$

At $(\pi, 0)$ we have two options for the direction of the small displacements $k_{n}$, and we find the best statistical precision with $\mathbf{K}_{0}+k_{n}=\left(\pi, k_{n}\right)$ and $(\pi-$ $\left.k_{n}, 0\right)$ for the triplet and singlet, respectively. In principle we can also define the velocity based solely on the gaps $\Delta_{\mu, \mathbf{K}_{0}+\mathrm{k}}$, but Eq. (35) has smaller size corrections. For $\mathbf{k} \neq 0$, we expect momentumdependent corrections in the form of integer powers of $1 / L[16]$,

$$
\Delta_{\mu, \mathbf{K}_{0}+\mathrm{k}}(L)=c k+B_{\mu, \mathbf{K}_{0}+\mathrm{k}} L^{-1}+O\left(L^{-2}\right),
$$

i.e., the nontrivial critical scaling behavior is seen only

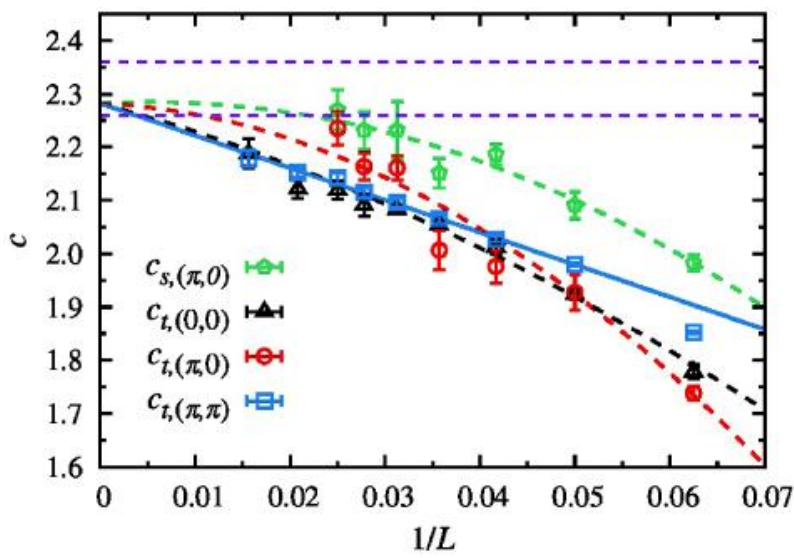

Fig. 22: (Fig. taken from Ref. 17). Size-dependent velocity estimators around the gapless points. The solid (blue) line is a fit to the $c_{t,(\pi, \pi)}$ data for $L=20$ and gives $c=2.282(5)\left(\chi^{2} / N_{\text {dof }} \approx 0.4\right)$. The fits to the other data sets use the same $c$ along with $L^{-1}$ and $L^{-2}$ corrections. The horizontal dashed lines indicate the value of $c \pm$ one standard deviation obtained using a winding-number estimator exactly at the gapless points $(\mathbf{k}=0)$, and the coefficients can be expanded as $B_{\mu, \mathbf{K}_{0}+\mathbf{k}}=a_{\mu, \mathbf{K}_{0}}+$ $b_{\mu, \mathbf{K}_{0}} k+O\left(k^{2}\right)$. Note that $\lim _{k \rightarrow 0} B_{\mu, \mathbf{K}_{0}+\mathrm{k}} \neq B_{\mu, \mathbf{K}_{0}}$ in general [16].

The estimator $c_{\mu, \mathbf{K}_{0}}(L)=c+b_{\mu, \mathbf{K}_{0}} / L+O\left(1 / L^{2}\right)$ converges to the correct velocity in the thermodynamic limit even at a critical point as long as $z=1$ [16]. We here analyze those singlets and triplets for which the gaps were determined to sufficient precision. As shown in Fig. 22, the velocities appear to converge to the same value. We have the highest precision for the triplet at $\mathbf{K}_{0}=(\pi, \pi)$, giving $c=2.282(5)$. For the other cases we simply fit curves with this $c$ fixed. The velocity estimates from the winding-number method and the direct gap measurements agree within statistical error. The velocities of the linearly dispersing modes for both singlets and triplets around all four gapless points being equal to each other again points to an emergent symmetry between the low-lying singlets and triplets at the critical point. We refer the readers to Ref. 17 for the discussion of other indicators of an enlarged $\mathrm{SO}(5)$ symmetry at the critical point based on the low-energy spectrum.

Thus, at the DQC point, there are both gapless singlets and triplets at $\mathbf{k}=(0,0),(\pi, 0),(0, \pi)$, and $(\pi$, p), forming eight gapless excitation modes, in total, with the same velocity, as illustrated in Fig. 23. The unique velocity for these gapless modes and the degenerate singlets and triplets clearly point to deconfined spinon excitations. From the fact that we
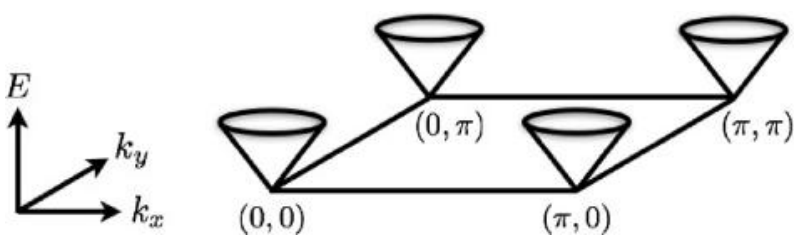

Fig. 23: (Fig. taken from Ref. 17). Schematic illustration of the low-lying energy spectrum at the deconfined quantum critical point. There are four gapless points for both $S=0$ and $S=1$ excitations, at $\mathbf{k}=(0$, $0),(\pi, 0),(0, \pi)$, and $(\pi, \pi)$. Close to these points the modes disperse linearly with the same velocity. The dispersion relation marks the lower edge of a continuum of excitations arising from two essentially deconfined spinons, with the singlespinon dispersion also being the same as the lower edge of the two-spinon continuum 
have measured two-spinon excitation from the ground state with $S=0$ and $\mathbf{k}=(0,0)$ via the projector algorithm which uniquely singles out the ground state with momentum $\mathbf{k}=(0,0)$, we infer that the singlespinon dispersion relation is equal to the degenerate $S$ $=0$ and $S=1$ dispersions at criticality (i.e., close to the $g=0.05$ curve in Fig. 19, which is already almost $L \rightarrow \infty$ converged away from the gapless points). This situation here is a direct analog of the excitations of the $S=1 / 2$ Heisenberg chain.

\section{Conclusions}

We have reviewed several methods to analyze the imaginary-time correlation functions generated using QMC techniques to extract the lowest singlet and triplet gaps for SU(2)-invariant antiferromagnets as a function of momentum at finite system sizes. The study of low-lying excitations provides a complementary point of view especially because such gaps may have significantly better convergence properties compared to conventionally measured

\section{References}

P Fazekas, Lecture Notes on Electron Correlation and Magnetism (World Scientific, 1999)

F Dyson, E H Lieb and B Simon (1978) J Stat Phys 86 335; E J Neves and J F Perez (1986) Phys Lett 114A, 331; T Kennedy, E H Lieb and B S Shastry J Stat Phys 531019 (1988)

J D Reger and A P Young (1988) Phys Rev B 375978

P Chandra and B Doucot Phys Rev B 389335 (1988); A V Chubukov Phys Rev B 44392 (1991); E. Dagotto and A Moreo Phys Rev Lett 632148 (1989); M P Gelfand et al. Phys Rev B 4010801 (1989)

L Wang and A W Sandvik, arXiv: 1702.08197v2

P W Anderson (1987) Science 2351196

S A Kivelson, D S Rokhsar and J P Sethna (1987) Phys Rev B 35 8865

N Read and S Sachdev (1991) Phys Rev Lett 661773

X G Wen (1991) Phys Rev B 442664

T Senthil and M P A Fisher (2000) Phys Rev B 627850

B S Shastry and B Sutherland (1981) Physica B 1081069

R R P Singh, M P Gelfand and D A Huse (1988) Phys Rev Lett 61 2484

S Sachdev Quantum Phase Transitions (Cambridge University Press, 2011) quantities in QMC studies such as correlation functions, order parameters etc. We have shown several applications of these methods for onedimensional and two-dimensional antiferromagnets which include extracting the velocity of propagating modes such as magnons, spinons and triplons, using level spectroscopy to locate the location of the quantum critical point and revealing the highly unusual excitation spectrum in the neighborhood of a deconfined quantum critical point in two dimensions. In conclusion, we expect these gap extraction procedures to be useful for the study of many other strongly correlated systems.

\section{Acknowledgments}

I would like to acknowledge my collaborators, Hidemaro Suwa and Anders W. Sandvik, as well as Debasish Banerjee, Kedar Damle, Pushan Majumdar and Roderich Moessner for useful discussions regarding various aspects of the work presented here.

M Troyer and U-J Wiese (2005) Phys Rev Lett 94170201

A W Sandvik AIP Conf Proc 1297, 135 (2010)

A Sen, H Suwa and A W Sandvik (2015) Phys Rev B 92195145

H Suwa, A Sen and A W Sandvik (2016) Phys Rev B 94144416

A W Sandvik (2005) Phys Rev Lett 95207203

A W Sandvik (1999) Phys Rev B 59 R14157

A W Sandvik and H G Evertz (2010) Phys Rev B 82024407

A W Sandvik (1992) J Phys A 253667

M Jarrell and J E Gubernatis (1996) Phys Rep 269133

H Suwa and S Todo (2015) Phys Rev Lett 115080601

J Des Cloizeaux and J J Pearson (1962) Phys Rev 1282131

R K Kaul and R G Melko (2008) Phys Rev B 78014417

F -J Jiang (2011) Phys Rev B 83024419

F -J Jiang and U -J Wiese (2011) Phys Rev B 83155120

E L Pollock and D M Ceperley (1987) Phys Rev B 368343

H Neuberger and T Ziman (1989) Phys Rev B 39 2608; D S Fisher (1989) ibid 39 11783; P Hasenfratz and F Niedermayer (1993) Z Phys B: Condes Matter 9291

P W Anderson (1952) Physical Review 86 694; C Lhuillier arXiv:Cond-mat/0502464; A Wietek, M Schuler, A M Lauchli, arXiv:1704.08622

C M Canali, S M Girvin and M Wallin (1992) Phys Rev B 45 10131 
O F Syljuasen and A W Sandvik (2002) Phys Rev E 66046701

R R P Singh, M P Gelfand and D A Huse (1988) Phys Rev Lett 61 2484

A J Millis and H Monien (1993) Phys Rev Lett 71 210; Phys Rev B 5016606 (1994)

A W Sandvik and D J Scalapino (1994) Phys Rev Lett 722777

L Wang, K S D Beach and A W Sandvik (2006) Phys Rev B 73 014431

A V Chubukov and D K Morr (1995) Phys Rev B 523521

M P A Fisher, P B Weichman, G Grinstein and D S Fisher (1989) Phys Rev B $\mathbf{4 0} 546$

A V Chubukov, S Sachdev and J Ye (1994) Phys Rev B 4911919

A W Sandvik, S Daul, R R P Singh and D J Scalapino (2002) Phys Rev Lett 89247201

O I Motrunich and A Vishwanath (2004) Phys Rev B 70075104

T Senthil, A Vishwanath, L Balents, S Sachdev and M P A Fisher (2004) Science $\mathbf{3 0 3} 1490$

T Senthil, L Balents, S Sachdev, A Vishwanath and M P A Fisher (2004) Phys Rev B 70144407

N Read and S Sachdev Phys Rev Lett 621694 (1989); Phys Rev B 424568 (1990)

A W Sandvik (2007) Phys Rev Lett 98227202

L Wang, Z-C Gu, F Verstraete and X-G Wen (2016) Phys Rev B 94075143

T Li, F Becca, W Hu and S Sorella (2012) Phys Rev B 86075111

S-S Gong, W Zhu, D N Sheng, O I Motrunich and M P A Fisher (2014) Phys Rev Lett 113027201

R G Melko and R K Kaul (2008) Phys Rev Lett 100017203

F J Jiang, M Nyfeler, S Chandrasekharan and U J Wiese J Stat Mech (2008) P02009
A W Sandvik (2010) Phys Rev Lett 104177201

R K Kaul (2011) Phys Rev B 84054407

K Harada, T Suzuki, T Okubo, H Matsuo, J Lou, H Watanabe, S Todo and N Kawashima (2013) Phys Rev B 88 220408(R)

M S Block, R G Melko and R K Kaul (2013) Phys Rev Lett 111 137202

S Pujari, F Alet and K Damle (2015) Phys Rev B 91104411

H Shao, W Guo and A W Sandvik (2016) Science 352213

A Nahum, P Serna, J T Chalker, M Ortuno and A M Somoza (2015) Phys Rev Lett 115267203

A B Kuklov, M Matsumoto, N V Prokofev, B V Svistunov and M Troyer (2008) Phys Rev Lett 101050405

K Chen, Y Huang, Y Deng, A B Kuklov, N V Prokofev and B V Svistunov (2013) Phys Rev Lett 110185701

K Nomura (1995) J Phys A: Math Gen 285451

S Eggert (1996) Phys Rev B 54 R9612

A W Sandvik (2010) Phys Rev Lett 104137204

E Manousakis (1991)Rev Mod Phys 631

R R P Singh and M P Gelfand (1995) Phys Rev B 52 R15695(R) 25

A W Sandvik and R R P Singh (2001) Phys Rev Lett 86528

M Powalski, G S Uhrig and K P Schmidt (2015) Phys Rev Lett 115207202

L Spanu, F Becca and S Sorella (2006) Phys Rev B 73134429

Y Huh, P Strack and S Sachdev (2013) Phys Rev Lett 111166401

E A Ghioldi, M G Gonzalez, L O Manuel and A E Trumper (2016) Europhys Lett 11357001. 